\title{
2d partition function in $\Omega$-background and vortex/instanton correspondence
}

\author{
Toshiaki Fujimori, ${ }^{a}$ Taro Kimura, ${ }^{a}$ Muneto Nitta ${ }^{a}$ and Keisuke Ohashi ${ }^{b}$ \\ ${ }^{a}$ Department of Physics, and Research and Education Center for Natural Sciences, Keio University, \\ Hiyoshi 4-1-1, Kanagawa 223-8521, Japan \\ ${ }^{b}$ Department of Physics, "E. Fermi", University of Pisa, \\ and INFN, Sezione di Pisa, \\ Largo Pontecorvo, 3, 56127 Pisa, Italy \\ E-mail: toshiaki.fujimori018@gmail.com, kimura@phys-h.keio.ac.jp, \\ nitta@phys-h.keio.ac.jp, keisuke084@gmail.com
}

ABSTRACT: We derive the exact vortex partition function in $2 \mathrm{~d} \mathcal{N}=(2,2)$ gauge theory on the $\Omega$-background, applying the localization scheme in the Higgs phase. We show that the partition function at a finite $\Omega$-deformation parameter $\epsilon$ satisfies a system of differential equations, which can be interpreted as a quantized version of the twisted $F$-term equations characterizing the SUSY vacua. Using the differential equations derived in this paper, we show the correspondence between the partition function of the two-dimensional vortex string worldsheet theory and the Nekrasov partition function at the root of Higgs branch of the four-dimensional $\mathcal{N}=2$ theory with two $\Omega$-deformation parameters $\left(\epsilon_{1}, \epsilon_{2}\right)$.

KEYwords: Supersymmetric gauge theory, Solitons Monopoles and Instantons, Field Theories in Lower Dimensions

ARXIV EPRINT: 1509.08630 


\section{Contents}

1 Introduction 1

2 Vortex partition function $\quad 3$

2.1 Omega background 3

$\begin{array}{lll}2.2 & \mathcal{Q} \text {-exact action and saddle points } & 6\end{array}$

2.3 One-loop determinants 8

$\begin{array}{lll}2.4 \mathcal{Q} \text {-closed operator } & 10\end{array}$

2.5 Vortex partition function and integral representations 13

$\begin{array}{lll}2.6 & \text { Generalization to non-Abelian gauge theories } & 15\end{array}$

3 Differential equation for the vortex partition function $\quad 19$

$\begin{array}{lll}3.1 & \mathrm{U}(1) \text { gauge theories with } N \text { chiral multiplets } & 19\end{array}$

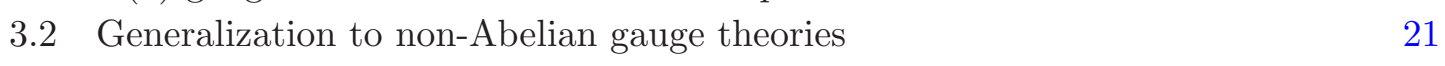

4 Vortex counting on vortex string worldsheet $\quad \mathbf{2 3}$

4.1 The vortex worldsheet theory: Hanany-Tong model 25

$\begin{array}{ll}4.2 & \text { Comparison with Nekrasov partition function } 28\end{array}$

5 Conclusion $\quad 32$

$\begin{array}{ll}\text { A Spinor conventions } & 32\end{array}$

B Mode expansion around saddle points $\quad 33$

B.1 Supermultiplets 34

B.2 Normalizability 36

\section{Introduction}

Recent progress on supersymmetric gauge theories allows us to perform path integrals in an explicit way through the localization scheme. Such exact results provide us with a number of non-trivial checks of gauge theory dualities, e.g. gauge/gravity duality, mirror symmetry, Seiberg duality. After the seminal work by Pestun [1], the localization scheme is widely applied to gauge theories on compact curved manifold, where the Infrared (IR) divergence is properly regularized by the curvature of the spacetime. On the other hand, the exact computation on a non-compact spacetime needs another way to deal with the IR divergence as in the earliest example of $\mathcal{N}=2$ supersymmetric gauge theories on the fourdimensional Euclidean spacetime [2]. One possible approach for the non-compact case is to consider the $\Omega$-deformed theories, which can be naturally obtained through the standard dimensional reduction from the corresponding higher dimensional theory in a non-trivial 
supersymmetric background. The exact partition function in $4 \mathrm{~d} \mathcal{N}=2$ gauge theories in the $\Omega$-background was computed in [2] and it was shown that the partition function reproduces the Seiberg-Witten prepotential, which captures the low-energy dynamics of the theories. Although, it cannot be applied to arbitrary dimensions, it allows us to perform the path integral in several physically important classes of theories.

In this paper, we revisit the $\Omega$-background in two dimensions and compute the exact vortex partition function [3-9], using the first-principles localization method in the Higgs phase, where the path integral localizes to BPS vortex configurations. We first show the supersymmetry transformation in the $\Omega$-deformed two-dimensional theory, and then apply the localization method based on the (scalar) supersymmetry $\mathcal{Q}$ preserved by the $\Omega$ background. To compute the one-loop determinant, we determine the bosonic and fermionic fluctuations around the BPS vortex configuration and count the short supermultiplets, which have non-trivial contribution to the partition function. Combining the one-loop determinant and the classical contribution from a certain $\mathcal{Q}$-closed operator, we obtain both the explicit (combinatorial) form of the vortex partition function, which can also be rewritten into an integral expression. As in the case of the four-dimensional theory, the whole partition function can be decomposed into the perturbative and the non-perturbative parts. The latter part is the contribution from the BPS vortices, which play a role of instantons in two dimensions [10-17]. We also show that the partition function does not simply factorize into two parts in the case involving fractional vortices.

Once the partition function in the $\Omega$-background is obtained, one can extract the nontrivial information for the low-energy dynamics of gauge theories by taking the limit where the $\Omega$-background is turned off, $\epsilon \rightarrow 0$. In addition to the role as the regulator, it is known that the $\Omega$-background parameter $\epsilon$ has another interesting interpretation as the quantum deformation parameter [18-20]. In this sense, the limit $\epsilon \rightarrow 0$ corresponds to the classical theory. Indeed the Seiberg-Witten curve, which appears in this limit, has an essential connection with the classical integrable systems. At this moment, the Seiberg-Witten curve is just an algebraic curve, which is characterized by an algebraic relation, $P(x, y)=$ 0 . Then, turning on the $\Omega$-background, the algebraic relation lifts up to a differential equation $P(\hat{x}, \hat{y}) \cdot \psi=0$, which is called the quantum curve. In our two-dimensional case, we show that the vortex partition function with finite $\epsilon$ satisfies a differential equation, where the corresponding differential operator is obtained by quantizing the twisted $F$ term equation, i.e. the algebraic relation characterizing the twisted superpotential. This differential equation allows us to examine the behavior of the partition function in the limit $\epsilon \rightarrow 0$ in an efficient way.

The $\mathcal{N}=(2,2)$ theory in two dimensions has various interesting connection with $\mathcal{N}=2$ theory in four dimensions. One of the important links between them is the correspondence between the BPS objects in two and four dimensions [21, 22]. ${ }^{1}$ This correspondence has been interpreted as the relation between the $2 \mathrm{~d}$ effective worldsheet theory on vortex strings and the parent $4 \mathrm{~d}$ gauge theory at the root of Higgs branch [29, 30]. In this paper we apply the exact formula to obtain the vortex partition function in the effective worldsheet theory

\footnotetext{
${ }^{1}$ See $[23-25]$ for recent progress along this direction, and also [26-28].
} 
of vortex strings, called the Hanany-Tong model [10]. We compare this vortex partition function with the four-dimensional Nekrasov partition function with two $\Omega$-deformation parameters $\left(\epsilon_{1}, \epsilon_{2}\right)$ and show the agreement of two and four-dimensional theories at the level of partition functions.

This paper is organized as follows. In section 2, we first reconsider the vortex partition function in the $\Omega$-background. Applying the localization technique in the Higgs phase, we derive the exact formula for the vortex partition function of Abelian $\mathcal{N}=(2,2)$ theories in two dimensions. Deriving the integral expression for the partition function, we show that the mirror symmetry can be naturally observed by rewriting the integral. We then generalize the result to the non-Abelian theory, and show that it coincides with the hemisphere partition function [31-33]. In section 3, we study the differential equation for the exact partition function. It is derived by a contour shift of the integral formula for the partition function, which can be viewed as a "quantized" version of the twisted $F$-term equation. In section 4, we study the vortex worldsheet theory, the Hanany-Tong model, as an application of the formula obtained in this paper. By comparing the differential equations for the partition functions, we obtain the agreement of two- and four-dimensional partition functions at the root of Higgs branch with two $\Omega$-deformation parameters $\left(\epsilon_{1}, \epsilon_{2}\right)$. In section 5 , we conclude this paper with some remarks.

\section{Vortex partition function}

In this section, we derive a formula for the vortex partition functions in $2 \mathrm{~d} \mathcal{N}=(2,2)$ supersymmetric theories in the $\Omega$-background. We apply the localization technique in the Higgs phase where the path integral localizes to BPS vortex configurations which are invariant under a certain symmetry transformation. Since $\Omega$-deformed $2 \mathrm{~d}$ theories can be obtained by means of the dimensional reduction from the corresponding $4 \mathrm{~d}$ theories with a non-trivial metric, we first consider $4 \mathrm{~d} \mathcal{N}=1$ theories and apply the localization technique to compute the vortex partition function.

\subsection{Omega background}

The 4-dimensional geometry corresponding to the $\Omega$-background takes the form

$$
d s^{2}=|d z-i z(\epsilon d w+\bar{\epsilon} d \bar{w})|^{2}+|d w|^{2},
$$

where $z=x_{1}+i x_{2}, w=x_{3}+i x_{4}$ and $x_{3}$ and $x_{4}$ directions are periodic: $x_{3} \sim x_{3}+2 \pi R_{3}$, $x_{4} \sim x_{4}+2 \pi R_{4}$. The $2 \mathrm{~d} \Omega$-background can be obtained by performing the dimensional reduction along the periodic directions $x_{3}$ and $x_{4}$. If we take the limit $\epsilon \rightarrow 0$, the geometry reduces to $\mathbb{R}^{2} \times T^{2}$ with the trivial metric and the resulting theory is the standard $2 \mathrm{~d}$ $\mathcal{N}=(2,2)$ model without any deformation. Let $e_{i}{ }^{\alpha}(\alpha=1,2,3,4)$ be the following basis for the vierbein

$$
d x^{i}\left(e_{i}{ }^{1}+i e_{i}{ }^{2}\right)=d z-i z(\epsilon d w+\bar{\epsilon} d \bar{w}), \quad d x^{i}\left(e_{i}^{3}+i e_{i}^{4}\right)=d w .
$$

Then, the spin connection takes the form (see appendix A for our conventions of spinors)

$$
\frac{1}{2} \omega_{i}^{\alpha \beta} \sigma_{\alpha \beta} d x^{i}=\frac{1}{2} \omega_{i}^{\alpha \beta} \bar{\sigma}_{\alpha \beta} d x^{i}=\frac{1}{2}(\epsilon d w+\bar{\epsilon} d \bar{w}) \sigma_{3} .
$$


The supersymmetry transformation in this geometry is parameterized by the parallel spinors $\varepsilon, \bar{\varepsilon}$ which satisfy the following equations

$$
\mathcal{D}_{i} \varepsilon=\left(\partial_{i}+\frac{i}{2} \omega_{i}^{\alpha \beta} \sigma_{\alpha \beta}-i V_{i}\right) \varepsilon=0, \quad \mathcal{D}_{i} \bar{\varepsilon}=\left(\partial_{i}+\frac{i}{2} \omega_{i}^{\alpha \beta} \bar{\sigma}_{\alpha \beta}+i V_{i}\right) \bar{\varepsilon}=0,
$$

where $V_{i}$ is a background $R$-symmetry gauge field. In order to have supersymmetries in the $\Omega$-deformed geometry, the background $R$-symmetry gauge field should be appropriately turned on so that the parallel spinor equations (2.4) have non-trivial solutions. If we assume that $V_{i}$ is given by

$$
V_{i} d x^{i}=-\frac{1}{2}(\epsilon d w+\bar{\epsilon} d \bar{w})
$$

the solutions of eq. (2.4) satisfying the periodic boundary condition are given by

$$
\varepsilon=\left(\begin{array}{c}
0 \\
\varepsilon_{0}
\end{array}\right), \quad \bar{\varepsilon}=\left(\begin{array}{c}
\bar{\varepsilon}_{0} \\
0
\end{array}\right),
$$

where $\varepsilon_{0}$ and $\bar{\varepsilon}_{0}$ are constants. Note that for these spinors, the spinor bilinear $\varepsilon \bar{\sigma}^{i} \bar{\varepsilon}$ is proportional to the Killing vector $\xi^{i}$ given by

$$
\frac{1}{2 \varepsilon_{0} \bar{\varepsilon}_{0}}\left(\varepsilon \bar{\sigma}^{i} \bar{\varepsilon}\right) \partial_{i}=\xi^{i} \partial_{i} \equiv \partial_{w}+i \epsilon\left(z \partial_{z}-\bar{z} \partial_{\bar{z}}\right)
$$

The field content of our model is the same as that of the standard $\mathcal{N}=1$ model in 4-dimensions. Furthermore, the supersymmetry variations of the fields with respect to the supercharge $\mathcal{Q} \equiv \varepsilon Q+\bar{\varepsilon} \bar{Q}$ also take the same form as those of $\mathcal{N}=1$ models if the background connections are appropriately incorporated into the covariant derivative. In the following, we first focus on the case of $\mathrm{U}(1)$ gauge theory for simplicity. The supersymmetry transformation for a vector multiplet is given by

$$
\begin{array}{rlrl}
\mathcal{Q} A_{i} & =-i\left(\bar{\lambda} \sigma_{i} \varepsilon-\bar{\varepsilon} \sigma_{i} \lambda\right), & \mathcal{Q} \lambda=-i F_{i j} \sigma^{i j} \varepsilon+D \varepsilon, \\
\mathcal{Q} D=i\left(\varepsilon \bar{\sigma}_{i} \mathcal{D}_{i} \bar{\lambda}+\mathcal{D}_{i} \lambda \bar{\sigma}_{i} \bar{\varepsilon}\right), & \mathcal{Q} \bar{\lambda}=i F_{i j} \bar{\sigma}^{i j} \bar{\varepsilon}+D \bar{\varepsilon},
\end{array}
$$

where the covariant derivative $\mathcal{D}_{i}$ contains the background $R$-symmetry gauge field

$$
\mathcal{D}_{i} \lambda=\left(\partial_{i}+\frac{i}{2} \omega_{i}^{\alpha \beta} \sigma_{\alpha \beta}-i V_{i}\right) \lambda, \quad \mathcal{D}_{i} \bar{\lambda}=\left(\partial_{i}+\frac{i}{2} \omega_{i}^{\alpha \beta} \bar{\sigma}_{\alpha \beta}+i V_{i}\right) \bar{\lambda} .
$$

For a chiral multiplet, the supersymmetry transformation is given by

$$
\begin{aligned}
\mathcal{Q} \phi & =\sqrt{2} \varepsilon \psi, & \mathcal{Q} \bar{\phi}=\sqrt{2} \bar{\varepsilon} \bar{\psi} \\
\mathcal{Q} \psi & =\sqrt{2}\left(i \mathcal{D}_{i} \phi \bar{\sigma}_{i} \bar{\varepsilon}+F \varepsilon\right), & \mathcal{Q} \bar{\psi}=\sqrt{2}\left(i \mathcal{D}_{i} \bar{\phi} \sigma_{i} \varepsilon+\bar{F} \bar{\varepsilon}\right), \\
\mathcal{Q} F=\sqrt{2}\left(i \mathcal{D}_{i} \psi \bar{\sigma}_{i} \bar{\varepsilon}+\sqrt{2} i \phi \bar{\varepsilon} \bar{\lambda}\right), & & \mathcal{Q} \bar{F}=\sqrt{2}\left(i \mathcal{D}_{i} \bar{\psi} \sigma_{i} \varepsilon+\sqrt{2} i \bar{\phi} \varepsilon \lambda\right) .
\end{aligned}
$$

Again, the covariant derivative contains the background gauge fields $V_{i}$ for the $R$-symmetry, under which a chiral multiplet $(\phi, \psi, F)$ with $R$-charge $q$ transforms as

$$
(\phi, \psi, F) \rightarrow e^{-i q \alpha}\left(\phi, e^{i \alpha} \psi, e^{2 i \alpha} F\right) .
$$


In addition to the $R$-symmetry gauge field background, we can also introduce a flat gauge field background $\tilde{A}_{i}$ for each U(1) subgroup of the Cartan part of the flavor symmetry

$$
\tilde{A}_{i}^{a} d x^{i}=m^{a} d w+\bar{m}^{a} d \bar{w} .
$$

The complex parameters $m^{a}$ are called the twisted masses in $2 \mathrm{~d} \mathcal{N}=(2,2)$ theories.

Assuming that $\varepsilon_{0}$ and $\bar{\varepsilon}_{0}$ are commuting parameters, we can show that the square of the supersymmetry closes to the translation along the compact directions $P_{w}$, the rotation $J$ in the $z$-plane, the $R$-symmetry $R$ and the flavor symmetries $F_{a}$

$$
\mathcal{Q}^{2}=4 \varepsilon_{0} \bar{\varepsilon}_{0}\left[P_{w}+\epsilon\left(J-\frac{1}{2} R\right)+m^{a} F_{a}\right]
$$

where $P_{w}$ should be understood as the covariant derivative including the gauge connection. If we redefine $\tilde{J} \equiv J-\frac{1}{2} R$ as a new generator for the rotation in the $z$-plane, the supercharges corresponding to the parameters $\varepsilon_{0}$ and $\bar{\varepsilon}_{0}$ can be regarded as scalars under $\tilde{J}$. Similarly, it is convenient to rewrite the spinor indices in terms of the new rotation generator $\tilde{J}$ to make the new rotational symmetry manifest

$$
\begin{array}{ll}
\left(\begin{array}{l}
\lambda_{1} \\
\lambda_{2}
\end{array}\right)=\left(\begin{array}{l}
\lambda_{z} \\
\lambda_{0}
\end{array}\right), \quad\left(\begin{array}{l}
\bar{\lambda}^{\mathrm{i}} \\
\bar{\lambda}^{\dot{2}}
\end{array}\right)=\left(\begin{array}{l}
\bar{\lambda}_{0} \\
\bar{\lambda}_{\bar{z}}
\end{array}\right), \\
\left(\begin{array}{l}
\psi_{1} \\
\psi_{2}
\end{array}\right)=\left(\begin{array}{l}
\psi_{0} \\
\psi_{\bar{z}}
\end{array}\right), & \left(\begin{array}{l}
\bar{\psi}^{\mathrm{i}} \\
\bar{\psi}^{\dot{2}}
\end{array}\right)=\left(\begin{array}{l}
\bar{\psi}_{z} \\
\bar{\psi}_{0}
\end{array}\right),
\end{array}
$$

and the auxiliary field in a chiral multiplet $(F, \bar{F})$ becomes $\left(F_{z}, \bar{F}_{\bar{z}}\right)$, where the quantities with the subscript 0 are scalars and $z, \bar{z}$ stand for $(1,0)$ and $(0,1)$-forms. It is also convenient to use the following notation

$$
\begin{aligned}
A_{z} & =\frac{1}{2} g^{i j}\left(e_{i}^{1}-i e_{i}^{2}\right) A_{j}, & A_{\bar{z}} & =\frac{1}{2} g^{i j}\left(e_{i}^{1}+i e_{i}^{2}\right) A_{j}, \\
A_{\xi} & =\frac{1}{2} g^{i j}\left(e_{i}^{3}-i e_{i}^{4}\right) A_{j}, & A_{\bar{\xi}} & =\frac{1}{2} g^{i j}\left(e_{i}^{3}+i e_{i}^{4}\right) A_{j},
\end{aligned}
$$

and similarly for the field strength $F_{i j}$. Note that $A_{\xi}$ and $A_{\bar{\xi}}$ can also be viewed as the gauge connection in the directions of the Killing vector fields $\xi=\partial_{w}+i \epsilon\left(z \partial_{z}-\bar{z} \partial_{\bar{z}}\right)$ and $\bar{\xi}=\partial_{\bar{w}}+i \bar{\epsilon}\left(z \partial_{z}-\bar{z} \partial_{\bar{z}}\right)$, that is

$$
A_{\xi}=\xi^{i} A_{i}, \quad A_{\bar{\xi}}=\bar{\xi}^{i} A_{i}
$$

In terms of these new notations, the supersymmetry transformation rules for a vector multiplet (2.8) and (2.9) can be rewritten as

$$
\begin{aligned}
\mathcal{Q} A_{z} & =-i \bar{\varepsilon}_{0} \lambda_{z}, & \mathcal{Q} A_{\xi} & =0, \\
\mathcal{Q} A_{\bar{z}} & =-i \varepsilon_{0} \bar{\lambda}_{\bar{z}}, & \mathcal{Q} A_{\bar{\xi}} & =i\left(\bar{\varepsilon}_{0} \lambda_{0}-\varepsilon_{0} \bar{\lambda}_{0}\right), \\
\mathcal{Q} \lambda_{z} & =-4 \varepsilon_{0} F_{\xi z}, & \mathcal{Q} \lambda_{0} & =\varepsilon_{0}\left(2 F_{z \bar{z}}+2 F_{\xi \bar{\xi}}+D\right), \\
\mathcal{Q} \bar{\lambda}_{\bar{z}} & =-4 \bar{\varepsilon}_{0} F_{\xi \bar{z}} & \mathcal{Q} \bar{\lambda}_{0} & =\bar{\varepsilon}_{0}\left(2 F_{z \bar{z}}-2 F_{\xi \bar{\xi}}+D\right), \\
\mathcal{Q} D & =2 i \mathcal{D}_{\xi}\left(\bar{\varepsilon}_{0} \lambda_{0}+\varepsilon_{0} \bar{\lambda}_{0}\right)-2 \mathcal{Q} F_{z \bar{z}}, & \mathcal{Q} F_{z \bar{z}} & =i\left(\bar{\varepsilon}_{0} \partial_{\bar{z}} \lambda_{z}-\varepsilon_{0} \partial_{z} \bar{\lambda}_{\bar{z}}\right),
\end{aligned}
$$


and the supersymmetry transformation rules for a chiral multiplet (2.11)-(2.13) become

$$
\begin{aligned}
\mathcal{Q} \phi & =\sqrt{2} \varepsilon_{0} \psi_{0}, & \mathcal{Q} \psi_{0} & =2 \sqrt{2} i \bar{\varepsilon}_{0} \mathcal{D}_{\xi} \phi, \\
\mathcal{Q} \bar{\phi} & =-\sqrt{2} \bar{\varepsilon}_{0} \bar{\psi}_{0}, & \mathcal{Q} \bar{\psi}_{0} & =-2 \sqrt{2} i \varepsilon_{0} \mathcal{D}_{\xi} \bar{\phi}, \\
\mathcal{Q} F_{\bar{z}} & =2 \bar{\varepsilon}_{0}\left(\sqrt{2} i \mathcal{D}_{\xi} \psi_{\bar{z}}-\sqrt{2} i \mathcal{D}_{\bar{z}} \psi_{0}-i \phi \bar{\lambda}_{\bar{z}}\right), & & \mathcal{Q} \psi_{\bar{z}}=\sqrt{2}\left(2 i \bar{\varepsilon}_{0} \mathcal{D}_{\bar{z}} \phi+\varepsilon_{0} F_{\bar{z}}\right), \\
\mathcal{Q} \bar{F}_{z} & =2 \varepsilon_{0}\left(\sqrt{2} i \mathcal{D}_{\xi} \bar{\psi}_{z}+\sqrt{2} i \mathcal{D}_{z} \bar{\psi}_{0}+i \bar{\phi} \lambda_{z}\right), & & \mathcal{Q} \bar{\psi}_{z}=\sqrt{2}\left(2 i \varepsilon_{0} \mathcal{D}_{z} \bar{\phi}+\bar{\varepsilon}_{0} \bar{F}_{z}\right),
\end{aligned}
$$

where $\mathcal{D}_{\xi}, \mathcal{D}_{\bar{\xi}}$ are the covariant derivatives containing all the connections. For example, for the fermionic field in the $a$-th flavor, $\mathcal{D}_{\xi}$ is given by

$$
\mathcal{D}_{\xi} \psi_{\bar{z}}^{a}=\left[\xi^{i} \partial_{i}+i A_{\xi}-i \epsilon+i\left(m_{a}+\frac{\epsilon q_{a}}{2}\right)\right] \psi_{\bar{z}}^{a} .
$$

In the following, we set $q_{a}=0$ since the $R$-charge, which appears in the combination $m_{a}+\epsilon q_{a} / 2$, can always be absorbed ${ }^{2}$ into the twisted mass $m_{a}$. The $R$-charge dependence can be restored by shifting the twisted mass $m_{a} \rightarrow m_{a}+\epsilon q_{a} / 2$.

\section{$2.2 \mathcal{Q}$-exact action and saddle points}

The vortex partition function can be defined by a path integral of the form

$$
Z=\int[\mathcal{D} \varphi] \exp (-\mathcal{Q} V+\mathcal{I})
$$

Here and in the following, $\mathcal{Q}$ denotes the supercharge with $\varepsilon_{0}=\bar{\varepsilon}_{0}=\frac{1}{2} . V$ and $\mathcal{I}$ are certain quantities satisfying

$$
\mathcal{Q}^{2} V=\mathcal{Q I}=0
$$

Then, we can show that $Z$ is invariant under any deformation $V \rightarrow V+\delta V$

$$
\delta Z=-\int[\mathcal{D} \varphi](\mathcal{Q} \delta V) \exp (-\mathcal{Q} V+\mathcal{I})=-\int[\mathcal{D} \varphi] \mathcal{Q}[\delta V \exp (-\mathcal{Q} V+\mathcal{I})]=0 .
$$

This property enables us to exactly evaluate the path integral $Z$ by using the deformation $V \rightarrow t V$ and applying the saddle point method in the limit $t \rightarrow \infty$.

Schematically, the $\mathcal{Q}$-exact part $\mathcal{Q} V$ can be expanded around its saddle points in terms of bosonic and fermionic fluctuations $\Phi$ and $\Psi$ :

$$
\mathcal{Q} V=\Phi^{\dagger} \Delta_{B} \Phi+\Psi^{\dagger} \Delta_{F} \Psi+\text { higher order terms, }
$$

where $\Delta_{B}$ and $\Delta_{F}$ are certain differential operators appearing in the linearized equations of motion around the saddle point configuration. The saddle point analysis implies that the vortex partition function can be written as a sum over the saddle points of $\mathcal{Q} V$

$$
Z=\sum\left[\exp (\mathcal{I}) \frac{\operatorname{det}\left(\Delta_{F}\right)}{\operatorname{det}\left(\Delta_{B}\right)}\right]_{\text {saddle points }} .
$$

\footnotetext{
${ }^{2}$ If we set $q=0$ for all chiral multiplets in the presence of a superpotential $W$, the twisted masses have to be assigned so that each term in $W$ has the total twisted mass $2 \times \frac{\epsilon}{2}=\epsilon$ since the total mass and the $R$-charge of $W$ should have $m=0, q=2$ in the original notation.
} 
Therefore, the exact vortex partition function $Z$ can be obtained by computing the contributions from each saddle points: the value of the $\mathcal{Q}$-closed operator $\mathcal{I}$ and the functional determinants of $\Delta_{B}$ and $\Delta_{F}$.

To discuss the explicit form of $\mathcal{Q} V$ and its saddle points, we restrict ourselves to the Abelian model with $N$ chiral multiplets with electric charge +1 . Let us consider the $\mathcal{Q}$-exact part generated from $V$ of the form

$$
\begin{aligned}
V= & \int d^{4} x\left[\frac{1}{g^{2}}\left(\lambda_{z} \overline{\mathcal{Q} \lambda_{z}}+\lambda_{0} \overline{\mathcal{Q} \lambda_{0}}+\bar{\lambda}_{0} \overline{\mathcal{Q} \bar{\lambda}_{0}}+\bar{\lambda}_{\bar{z}} \overline{\mathcal{Q} \bar{\lambda}_{\bar{z}}}\right)\right. \\
& \left.+\psi_{0}^{a} \overline{\mathcal{Q} \psi_{0}^{a}}+\psi_{\bar{z}}^{a} \overline{\mathcal{Q} \psi_{\bar{z}}^{a}}+\bar{\psi}_{z}^{a} \overline{\mathcal{Q} \bar{\psi}_{z}^{a}}+\bar{\psi}_{0}^{a} \overline{\mathcal{Q} \bar{\psi}_{0}^{a}}+i\left(\lambda_{0}+\bar{\lambda}_{0}\right)\left(\left|\phi^{a}\right|^{2}-r\right)\right]
\end{aligned}
$$

where $g$ and $r$ are constants corresponding to the gauge coupling constant and FayetIliopoulos (FI) parameter. Note that the vortex partition function do not depend on such parameters contained in the $\mathcal{Q}$-exact part. For eq. (2.37), $\mathcal{Q} V$ takes the form

$$
\mathcal{Q} V=S_{4 d}-2 i r \int d^{4} x F_{z \bar{z}}+\{\text { total derivative terms }\}
$$

where $S_{4 d}$ is the following action which reduces to that of the standard $\mathcal{N}=1$ supersymmetric model in the limit $\epsilon \rightarrow 0$ :

$$
\begin{aligned}
S_{4 d}= & \int d^{4} x\left[\frac{1}{4 g^{2}} F_{i j} F^{i j}+\frac{1}{2 g^{2}} D^{2}-\frac{i}{2 g^{2}} \bar{\lambda} \sigma^{i} \stackrel{\leftrightarrow}{\mathcal{D}}_{i} \lambda+\mathcal{D}_{i} \phi_{a} \mathcal{D}^{j} \bar{\phi}_{a}+\left|F_{a}\right|^{2}\right. \\
& \left.-\frac{i}{2} \bar{\psi}_{a} \sigma^{i} \stackrel{\leftrightarrow}{\mathcal{D}}_{i} \psi_{a}-\sqrt{2} i\left(\phi_{a} \bar{\psi}_{a} \bar{\lambda}+\bar{\phi}_{a} \psi_{a} \lambda\right)+i D\left(\left|\phi_{a}\right|^{2}-r\right)\right] .
\end{aligned}
$$

Note that the other terms in $\mathcal{Q} V$ are topological and total derivative terms. After integrating out the auxiliary fields $F_{a}$ and $D$, the bosonic part of $\mathcal{Q} V$ takes the form

$$
\begin{aligned}
(\mathcal{Q} V)_{\text {bosonic }}= & \int d^{4} x\left[\frac{1}{2 g^{2}}\left\{\left|2 i F_{z \bar{z}}+e^{2}\left(\left|\phi_{a}\right|^{2}-r\right)\right|^{2}+\left|F_{\xi \bar{\xi}}\right|^{2}+8\left|F_{\xi z}\right|^{2}+8\left|F_{\xi \bar{z}}\right|^{2}\right\}\right. \\
& \left.+4\left|\mathcal{D}_{\bar{z}} \phi_{a}\right|^{2}+2\left|\mathcal{D}_{\xi} \phi_{a}\right|^{2}+2\left|\mathcal{D}_{\xi} \bar{\phi}_{a}\right|^{2}\right] .
\end{aligned}
$$

This completed square form implies that the saddle points of $\mathcal{Q} V$ are the solutions of the following BPS equations

$$
\begin{aligned}
\mathcal{D}_{\bar{z}} \phi_{a} & =0, \quad 2 i F_{z \bar{z}}+g^{2}\left(\left|\phi_{a}\right|^{2}-r\right)=0, \\
F_{\xi \bar{\xi}} & =F_{\xi z}=F_{\xi \bar{z}}=\mathcal{D}_{\xi} \phi_{a}=\mathcal{D}_{\xi} \bar{\phi}_{a}=0 .
\end{aligned}
$$

The first two equations do not depend on the deformation parameter $\epsilon$ and can be identified with the BPS vortex equations in the corresponding $\mathcal{N}=(2,2)$ model without any deformation. The solution to eqs. (2.41) can be formally written as

$$
A_{\bar{z}}=-\frac{i}{2} \partial_{\bar{z}} \omega, \quad \phi_{a}=\sqrt{r} e^{-\frac{1}{2} \omega} h_{a}(z)
$$


where $h_{a}(z)$ are arbitrary holomorphic polynomials of $z$. The profile function $\omega$ is the solution of the following equation $[13,15]$

$$
\partial_{z} \partial_{\bar{z}} \omega=\frac{g^{2} r}{2}\left(1-H_{0} H_{0}^{\dagger} e^{-\omega}\right)
$$

where $H_{0}(z) \equiv\left(h_{1}, h_{2}, \cdots, h_{N}\right)$ and the boundary condition is $\lim _{|z| \rightarrow \infty} \omega=\log \left(H_{0} H_{0}^{\dagger}\right)$.

In the undeformed massless theory $\left(m_{a}=\epsilon=0\right)$, each component of $H_{0}(z)$ can be an arbitrary polynomial, whose coefficients are regarded as the moduli parameters of the BPS vortex configurations. On the other hand, in the presence of the twisted mass and the omega deformation, eqs. (2.42) tells us that $H_{0}(z)$ is restricted to the fixed points of the bosonic symmetry generated by

$$
\mathcal{Q}^{2}=P_{w}+\epsilon(J-R / 2)+m^{a} F_{a}
$$

Such configurations are specified by the vortex number $k \in \mathbb{Z}_{\geq 0}$ and the flavor label $a \in(1,2, \cdots, N)$ which specifies the vacuum at the spatial infinity. For the $k$-vortex configuration in the $a$-th vacuum, the components of $H_{0}(z)$ are given by

$$
h_{a}=z^{k}, \quad h_{b}=0 \quad(b \neq a),
$$

and eqs. (2.42) is satisfied if

$$
A_{\xi}=-m_{a}-k \epsilon
$$

In conclusion, the saddle point configuration specified by the vortex number $k$ and the vacuum label $a$ is given by

$$
A_{\xi}=-m_{a}-k \epsilon, \quad A_{\bar{z}}=-\frac{i}{2} \partial_{\bar{z}} \omega, \quad \phi_{b}=\left\{\begin{array}{cl}
\sqrt{r} e^{-\frac{1}{2} \omega} z^{k} & \text { for } b=a \\
0 & \text { for } b \neq a
\end{array} .\right.
$$

Although the solution of eq. (2.44) is not known, the vortex partition function $Z$ can be determined without the explicit use of the profile function $\omega$.

\subsection{One-loop determinants}

Before specifying the $\mathcal{Q}$-closed operator $\mathcal{I}$ for the vortex partition function, let us first discuss the functional determinants in eq. (2.36). For each saddle point configuration, the determinants can be calculated by finding the eigenmodes of the differential operators $\Delta_{B}$ and $\Delta_{F}$. (see appendix B for the details of the computations).

Since the saddle points are BPS configurations preserving the supersymmetry, the bosonic and fermionic fluctuations around them form supermultiplets. Generic supermultiplets do not contribute to the ratio of the determinants since they consist of one bosonic mode with eigenvalue $\Delta_{B} \doteq m_{+} m_{-}$and two fermionic modes with $\Delta_{F} \doteq m_{ \pm}$.

Besides such generic supermultiplets, there exist short supermultiplets which consist of one bosonic and one fermionic modes, so that they have non-trivial contributions to the 
determinants. As shown in the appendix B, their eigenvalues of $\Delta_{B}$ and $\Delta_{F}$ are related to those of the covariant derivative $\mathcal{D}_{\xi}$ in the following way:

$$
\Delta_{B} \doteq-\mathcal{D}_{\xi} \mathcal{D}_{\bar{\xi}}, \quad \Delta_{F} \doteq-i \mathcal{D}_{\bar{\xi}}
$$

Therefore, the ratio of the determinants can be obtained from the determinant of $\mathcal{D}_{\bar{\xi}}$ restricted to the short supermultiplets

$$
\left.\frac{\operatorname{det} \Delta_{F}}{\operatorname{det} \Delta_{B}} \propto \frac{1}{\operatorname{det}\left(-i \mathcal{D}_{\xi}\right)}\right|_{\text {short }} .
$$

As shown in appendix B, the bosonic parts of such short multiplets are given by the solutions of the linearized version of the BPS vortex equations (2.41) in the undeformed theory. They can be obtained by linearizing the general form of the vortex solution eq. (2.43) around the saddle point configuration

$$
\delta A_{\xi}=0, \quad \delta A_{\bar{z}}=-\frac{i}{2} \partial_{\bar{z}} \delta \omega, \quad \delta \phi_{b}=\sqrt{r} e^{-\frac{1}{2} \omega}\left[\delta h_{b}(z)-\frac{1}{2} \delta \omega h_{b}(z)\right],
$$

where $\delta h_{b}(z)$ are arbitrary holomorphic functions and once they are given, the function $\delta \omega$ can be uniquely determined through the linearized version of eq. (2.44) (see eq. (B.24)).

Although $\delta h_{b}(z)$ are arbitrary, not all degrees of freedom contained in $\delta h_{b}(z)$ are physical since the solution eq. (2.51) is invariant under the following infinite dimensional transformation:

$$
\delta h_{b}(z) \rightarrow \delta h_{b}(z)+v(z) h_{b}(z), \quad \delta \omega \rightarrow \delta \omega+2 v(z),
$$

where $v(z)$ is an arbitrary polynomial. Since $h_{a}(z)=z^{k}$ and $h_{b}(z)=0(b \neq a)$ for the $k$-vortex background in the $a$-th vacuum, only $\delta h_{a}(z)$ varies under this transformation. In order to find out the physical degrees of freedom, it is convenient to expand $\delta h_{b}(z)$ and fix the redundancy eq. (2.52) by truncating the sum in $h_{a}(z)$ in the following way:

$$
\delta h_{a}(z)=\sum_{l=0}^{k-1} c_{a, l} z^{l}, \quad \delta h_{b}(z)=\sum_{l=0}^{\infty} c_{b, l} z^{l} \quad(b \neq a) .
$$

where the coefficients $c_{b . l}$ are periodic functions of $x_{3}$ and $x_{4}$, so that they can be further expanded into the Kaluza-Klein modes

$$
c_{b, l}\left(x_{3}, x_{4}\right)=\sum_{\mathbf{n}} c_{b, l}^{\mathbf{n}} \exp i\left(\frac{n_{3} x_{3}}{R_{3}}+\frac{n_{4} x_{4}}{R_{4}}\right), \quad \mathbf{n} \equiv\left(n_{3}, n_{4}\right)
$$

There is a caveat here. It is well-known that the modes with $l \geq k-1(b \neq a)$ are non-normalizable in the $k$-vortex background. In general, if there exists a non-normalizable mode, the spectrum is continuous and the boson-fermion cancellation argument may not be applicable. However, we can show that there exists a continuous deformation of the $\mathcal{Q}$ exact part of the action under which all the modes become normalizable (see appendix B.2). Therefore, there is no need to care about the normalizability of the eigenmodes and the determinant can be calculated by using eq. (2.50). 
The eigenvalue of $-i \mathcal{D}_{\xi}$ can be easily read off by noting that its action on $\Delta \phi_{b}$ reduces to the following action on $c_{b, l}^{\mathbf{n}}$ :

$$
-i \mathcal{D}_{\xi} \phi_{b} \rightarrow \lambda_{b, l}^{\mathbf{n}} c_{b, l}^{\mathbf{n}} \equiv\left[-\frac{i}{2}\left(\frac{n_{3}}{R_{3}}-i \frac{n_{4}}{R_{4}}\right)+m_{b}-m_{a}+\epsilon(l-k)\right] c_{b, l}^{\mathbf{n}} .
$$

The functional determinants can be obtained by taking the product of the eigenvalues $\lambda_{b, l}^{\mathbf{n}}$ for all the physical eigenmodes

$$
\left[\frac{\operatorname{det} \Delta_{F}}{\operatorname{det} \Delta_{B}}\right]_{4 d, a, k}=\prod_{\mathbf{n}}\left[\left(\prod_{l=0}^{k-1} \frac{\Lambda_{0}}{\lambda_{a, l}^{\mathbf{n}}}\right) \prod_{b \neq a} \prod_{l=0}^{\infty} \frac{\Lambda_{0}}{\lambda_{b, l}^{\mathbf{n}}}\right],
$$

where we have introduced a scale parameter $\Lambda_{0}$ so that the ratio of the determinants is dimensionless. Now let us focus on the $2 \mathrm{~d}$ limit $R_{3}, R_{4} \rightarrow 0$, in which all the Kaluza Klein modes decouple and their contributions become trivial. Then, the one-loop determinant in two dimension can be obtained by ignoring the modes with $\left(n_{3}, n_{4}\right) \neq 0$

$$
\left[\frac{\operatorname{det} \Delta_{F}}{\operatorname{det} \Delta_{B}}\right]_{2 d, a, k}=\frac{(-1)^{k}}{k !}\left(\frac{\Lambda_{0}}{\epsilon}\right)^{N\left(\frac{m_{a}}{\epsilon}+k\right)} \prod_{b \neq a} \sqrt{\frac{\Lambda_{0}}{2 \pi \epsilon}} \Gamma\left(\frac{m_{b}-m_{a}-k \epsilon}{\epsilon}\right),
$$

where we have assumed $\sum_{b=1}^{N} m_{b}=0$ (this can always be done by shifting the scalar field $\sigma$ in the $2 \mathrm{~d}$ vector multiplet) and the infinite products are reduced to the gamma functions by using the zeta function regularization ${ }^{3}$

$$
\prod_{l=0}^{\infty} \frac{y}{z+l}=\exp \left[\lim _{s \rightarrow 0} \frac{\partial}{\partial s}\left\{y^{s} \zeta(s, z)\right\}\right]=y^{-z} \sqrt{\frac{y}{2 \pi}} \Gamma(z) .
$$

\section{$2.4 \mathcal{Q}$-closed operator}

Let us next turn to the $\mathcal{Q}$-closed part $\mathcal{I}$. In the following, we will work in two dimensions unless otherwise stated. In order to write down $\mathcal{I}$, it is convenient to use the twisted chiral superfield for the vector multiplet, which is expressed in terms of the anti-commuting Grassmann coordinates $\theta_{z}$ and $\bar{\theta}_{\bar{z}}$ as ${ }^{4}$

$$
\Sigma=\sigma+\frac{1}{2} \lambda_{z} \theta_{\bar{z}}+\frac{1}{2} \bar{\lambda}_{\bar{z}} \bar{\theta}_{z}+i F_{z \bar{z}} \theta_{\bar{z}} \bar{\theta}_{z}
$$

where $\sigma$ is the complex scalar field obtained from $A_{w}$ by the dimensional reduction. The supercharge which generates the transformation eqs. (2.22)-(2.26) is now expressed as the following differential operator acting on twisted chiral superfields:

$$
\mathcal{Q}=\theta_{\bar{z}} \frac{\partial}{\partial z}+\bar{\theta}_{z} \frac{\partial}{\partial \bar{z}}+\epsilon\left(z \frac{\partial}{\partial \theta_{\bar{z}}}-\bar{z} \frac{\partial}{\partial \bar{\theta}_{z}}\right) .
$$

\footnotetext{
${ }^{3}$ The Hurwitz zeta function $\zeta(s, z)=\sum_{l=0}^{\infty}(z+l)^{-s}$ satisfies

$$
\zeta(0, z)=-z+\frac{1}{2}, \quad \lim _{s \rightarrow 0} \frac{\partial}{\partial s} \zeta(s, z)=\log \frac{\Gamma(z)}{\sqrt{2 \pi}} .
$$

${ }^{4}$ Although this twisted chiral superfield $\Sigma$ is different from the one used in the standard $\mathcal{N}=(2,2)$ models, the difference vanishes when it is evaluated at the saddle points.
} 
With the identification $\theta_{\bar{z}}=d z, \bar{\theta}_{z}=d \bar{z}$, this differential operator can be identified with the equivariant exterior derivative with the equivariant parameter $\epsilon$ for the $\mathrm{SO}(2)$ rotation on the $z$-plane.

Let $\widetilde{W}$ be a twisted superpotential, i.e. a holomorphic function of twisted chiral superfields. Since the supersymmetry transformation $\mathcal{Q}$ acts on $\widetilde{W}$ as a total derivative, we can obtain a $\mathcal{Q}$-closed operator by integrating $\widetilde{W}$ over the superspace:

$$
\mathcal{I}=2 \int d^{2} x d \bar{\theta}_{\bar{z}} d \theta_{z} \widetilde{W}(\Sigma)
$$

Let $\tau_{0}$ be the following combination of the theta angle and the FI parameter:

$$
\tau_{0} \equiv \frac{\theta}{2 \pi}+i r
$$

The $\mathcal{Q}$-closed operator corresponding to the vortex partition function can be obtained from the standard tree-level twisted superpotential $\widetilde{W}=i \tau_{0} \Sigma$ by promoting the constant parameter $\tau_{0}$ to a background twisted chiral superfield $T$ :

$$
\widetilde{W}=i T(\alpha) \Sigma,
$$

where we have assumed that $T$ is a function of the following "equivariantly closed form":

$$
\alpha=\epsilon\left(\theta_{\bar{z}} \bar{\theta}_{z}+\epsilon|z|^{2}\right), \quad \mathcal{Q} \alpha=0 .
$$

In addition, we also assume that $T$ is a function satisfying

$$
T(0)=\tau_{0}, \quad \lim _{x \rightarrow \infty} T(x)=0 .
$$

For instance, $T(\alpha)=\tau_{0} e^{-\alpha}$ is a typical example. As long as these conditions are satisfied, the explicit form of $T(\alpha)$ is not essential since different choices of $T$ lead to $\mathcal{Q}$-closed operators which differ only by $\mathcal{Q}$-exact terms. The twisted superpotential preserves the supersymmetry since the background twisted chiral superfield $T(\alpha)$ is $\mathcal{Q}$-closed. For this choice of the twisted superpotential, the corresponding $\mathcal{Q}$-closed operator $\mathcal{I}$ is given by

$$
\mathcal{I}=\int d^{2} z 2 i\left[\epsilon T^{\prime}\left(|\epsilon z|^{2}\right) \sigma+i T\left(|\epsilon z|^{2}\right) F_{z \bar{z}}\right]=\int d^{2} z 2 i \epsilon T^{\prime}\left(|\epsilon z|^{2}\right)\left[\sigma+i \epsilon\left(z A_{z}-\bar{z} A_{\bar{z}}\right)\right],
$$

where we have used $\lim _{x \rightarrow \infty} T(x)=0$. This quantity can be obtained from the following gauge invariant quantity in the $4 \mathrm{~d}$ theory:

$$
\mathcal{I}_{4 d}=\frac{1}{(2 \pi)^{2} R_{3} R_{4}} \int d^{4} x 2 i \epsilon T^{\prime}\left(|\epsilon z|^{2}\right) A_{\xi}
$$

Therefore, at the saddle point eq. (2.48), $\mathcal{I}$ takes the value

$$
\left.\mathcal{I}\right|_{a, k}=\frac{2 \pi}{\epsilon} \int_{0}^{\infty} d|\epsilon z|^{2} i T^{\prime}\left(|\epsilon z|^{2}\right)\left(-m_{a}-k \epsilon\right)=2 \pi i\left(\frac{m_{a}}{\epsilon}+k\right) \tau_{0}
$$

where we have used $T(0)=\tau_{0}$. 
$\boldsymbol{Z}\left(\boldsymbol{\tau}_{\mathbf{0}}\right)$ as a generating function. The supersymmetry transformation rules (2.22)-(2.26) imply that in the limit $\epsilon \rightarrow 0$, the scalar field $\sigma$ becomes a $\mathcal{Q}$-closed operator whose difference at two arbitrary points is $\mathcal{Q}$-exact

$$
\left.\sigma\right|_{z=z_{1}}-\left.\sigma\right|_{z=z_{2}}=\frac{1}{2} \int_{z_{2}}^{z_{1}} \mathcal{Q}\left(\lambda_{z} d z+\lambda_{\bar{z}} d \bar{z}\right)+\mathcal{O}(\epsilon)
$$

Thus, we can ignore the position dependence of $\sigma$ in the limit $\epsilon \rightarrow 0$, so that the leading order term in $\mathcal{I}$ is given by

$$
\mathcal{I}=\int d^{2} z 2 i T^{\prime}\left(\epsilon|z|^{2}\right)[\sigma+\mathcal{O}(\epsilon)] \sim-\frac{2 \pi i \tau_{0}}{\epsilon}[\sigma+\mathcal{O}(\epsilon)]
$$

where $\sim$ denotes the equality up to $\mathcal{Q}$-exact terms. Since $\mathcal{Q}$-exact terms do not contribute to the path integral, eq. (2.70) implies that the expectation value of $\sigma$ can be calculated from $Z\left(\tau_{0}\right)$ in the following way ${ }^{5}$

$$
\langle\sigma\rangle=\lim _{\epsilon \rightarrow 0}\left(-\frac{\epsilon}{2 \pi i} \frac{\partial}{\partial \tau_{0}}\right) \log Z
$$

In the limit $\epsilon \rightarrow 0$, the twisted superpotential reduces to the standard one $\widetilde{W}=i \tau_{0} \Sigma$, and hence the action $-\mathcal{Q} V+\mathcal{I}$ becomes that of the conventional $\mathcal{N}=(2,2)$ model with the topological $\theta$ term

$$
-\mathcal{Q} V+\mathcal{I}=-S_{2 d}-\frac{i \theta}{2 \pi} \int F+\{\text { total derivative }\}
$$

where $S_{2 d}$ is the $2 \mathrm{~d}$ action obtained from eq. (2.39) by the dimensional reduction. Therefore, $\langle\sigma\rangle$ is the expectation value in the standard $\mathcal{N}=(2,2)$ model. It can also be obtained from the on-shell effective twisted superpotential:

$$
\langle\sigma\rangle=-i \partial_{\tau_{0}} \widetilde{W}_{\mathrm{eff}}
$$

Comparing with eq. (2.71), we find that the on-shell effective twisted superpotential and $Z$ are related by

$$
\widetilde{W}_{\text {eff }}\left(\tau_{0}\right)=-\lim _{\epsilon \rightarrow 0} \frac{\epsilon}{2 \pi} \log Z\left(\tau_{0}\right)
$$

The off-shell effective twisted superpotential for $\sigma$ can be obtained from $\widetilde{W}_{\text {eff }}\left(\tau_{0}\right)$ by the integrating-in procedure, i.e. the Legendre transformation. We will use it in the next section to check that our vortex partition function correctly reproduces the known results in $\mathcal{N}=(2,2)$ models.

\footnotetext{
${ }^{5}$ Note that the FI term in $\mathcal{Q} V$ does not contribute to eq. (2.71) since $Z$ does not depend on the parameters contained in the $\mathcal{Q}$-exact part.
} 


\subsection{Vortex partition function and integral representations}

Having obtained the one-loop determinant eq. (2.57) and the $\mathcal{Q}$-closed part eq. (2.68), we can obtain the vortex partition function from the saddle point formula eq. (2.36) by summing over the saddle points. Since $z$-plane is non-compact, the vacuum at spatial infinity has to be fixed and hence the vortex partition function is defined for each vacuum. Summing over the vortex number $k$ in the $a$-th vacuum, we obtain the following form of the vortex partition function:

$$
Z_{a}=\sum_{k=0}^{\infty} Z_{a, k}=\exp \left(\frac{2 \pi i m_{a} \tau}{\epsilon}\right) \sum_{k=0}^{\infty} e^{2 \pi i k \tau} \frac{(-1)^{k}}{k !} \prod_{b \neq a} \Gamma\left(\frac{m_{b}-m_{a}-k \epsilon}{\epsilon}\right) .
$$

where we have ignored the overall factor $\left(\Lambda_{0} / \epsilon\right)^{\frac{N-1}{2}}$ and the UV parameter $\tau_{0}$ is replaced with renormalized parameter $\tau$ defined at $\mu=\epsilon$

$$
2 \pi i \tau=2 \pi i \tau_{0}+\log \frac{\Lambda_{0}^{N}}{\epsilon^{N}} .
$$

It is worth pointing out that the ratio of $Z_{a, k}$ and $Z_{a, 0}$ agrees with the following finite dimensional integral on the vortex moduli space

$$
\frac{Z_{a, k}}{Z_{a, 0}}=e^{2 \pi i k \tau} \int_{\mathcal{M}_{a, k}} \exp \left(-S_{\mathrm{eff}, a, k}\right)=e^{2 \pi i k \tau} \prod_{b=1}^{N} \prod_{j=1}^{k} \frac{\epsilon}{m_{b}-m_{a}-j \epsilon},
$$

where $S_{\text {eff }, a, k}$ is the $\mathcal{Q}$-exact vortex effective action. This implies that $Z_{a, k}(k=0,1, \cdots)$ have a common irrational part, and hence the vortex partition function eq. (2.75) factorizes as

$$
Z_{a}=Z_{a}^{\mathrm{pert}} Z_{a}^{\mathrm{vort}}
$$

where the perturbative part $Z_{a}^{\text {pert }} \equiv Z_{a, 0}$ and the vortex part $Z_{a}^{\text {vort }} \equiv \sum_{k=0}^{\infty} Z_{a, k} / Z_{a, 0}$ are given by

$$
Z_{a}^{\text {pert }}=e^{\frac{2 \pi i m_{a} \tau}{\epsilon}} \Gamma\left(\frac{m_{b}-m_{a}}{\epsilon}\right), \quad Z_{a}^{\text {vort }}={ }_{0} F_{N-1}\left(\left\{1-\frac{m_{b}-m_{a}}{\epsilon}\right\}_{b \neq a},(-1)^{N} e^{2 \pi i \tau}\right) .
$$

The factorization eq. (2.78) is a general property of the vortex partition function except when there is contribution from the so-called fractional vortices (see section 2.6).

We can also rewrite the result eq. (2.75) into an integral form which will be more convenient in the discussion below. Since the gamma function $\Gamma(x)$ has poles at $x=-k \in$ $\mathbb{Z}_{\leq 0}$ with residue $(-1)^{k} / k$ ! , the infinite sum over the vortex number $k$ can be rewritten into the following contour integral along a path surrounding all the poles of the gamma function

$$
\sum_{k=0}^{\infty} \frac{(-1)^{k}}{k !} f(-k)=\int \frac{d x}{2 \pi i} \Gamma(x) f(x)
$$




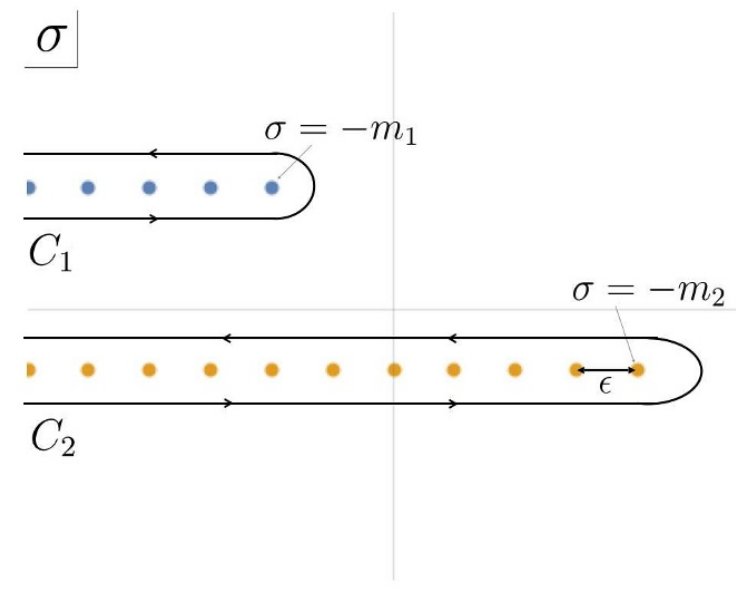

Figure 1. The contours in the complex $\sigma$-plane $(\epsilon \in \mathbb{R}, \epsilon>0)$. The integration along $C_{a}$ gives the vortex partition function in the $a$-th vacuum.

Applying this formula to eq. (2.75) and changing the integration variable ${ }^{6} \sigma=\epsilon x-m_{a}$, we obtain the integral form of the vortex partition function

$$
Z_{a}=\int_{C_{a}} \frac{d \sigma}{2 \pi i \epsilon} \exp \left(-\frac{2 \pi i \sigma \tau}{\epsilon}\right) \prod_{b=1}^{N} \Gamma\left(\frac{\sigma+m_{b}}{\epsilon}\right),
$$

Note that the integrand is independent of the vacuum label $a$, so that only the integration contour has the information on the choice of vacuum. The vortex partition function in the $a$-th vacuum can be obtained by choosing a contour which encloses all the poles of $a$-th Gamma function (see figure 1).

A dual description. As is well-known, our model has the mirror dual description in which the dynamical degrees of freedom are described by twisted chiral multiplets [34]. The vortex partition function can also be expressed in terms of the twisted superpotential in the dual theory. In the case of $\mathrm{U}(1)$ gauge theory with $N$ charged chiral multiplets, the integral form of the vortex partition function eq. (2.81) can be rewritten into the following form

$$
Z_{a}=\int \frac{d \sigma}{2 \pi i \epsilon} d y_{1} \cdots d y_{N} \exp \left(-\frac{2 \pi}{\epsilon} \widetilde{W}\right)
$$

where $\widetilde{W}$ is the following twisted superpotential in the dual theory:

$$
\widetilde{W}=i \sigma \tau-\frac{1}{2 \pi} \sum_{a=1}^{N}\left[\left(\sigma+m_{b}\right) y_{a}-\epsilon e^{-y_{a}}\right] .
$$

\footnotetext{
${ }^{6}$ The new integration variable is denoted by $\sigma$ as the position of each pole is given by $\left.\sigma\right|_{z=0}$ in the corresponding saddle point configuration:

$$
\left.\sigma\right|_{z=0}=\left[-m_{a}-k \epsilon+\frac{1}{2} \epsilon\left(z \partial_{z}+\bar{z} \partial_{\bar{z}}\right) \omega\right]_{z=0}=-m_{a}-k \epsilon .
$$
}


Noting that each integral over $y_{a}$ gives the gamma function

$$
\Gamma(x)=\int_{-\infty}^{\infty} d y \exp \left(x y-e^{y}\right),
$$

we can easily see that this integral expression (2.82) reduces to eq. (2.81). The existence of the integral representation of $Z$ in terms of the dual twisted superpotential implies that eq. (2.82) can be directly obtained as a result of the localization computation in the $\Omega$-deformed mirror model.

\subsection{Generalization to non-Abelian gauge theories}

Our result can be easily generalized to non-Abelian theories with arbitrary gauge groups and matter representations. Let us consider a model with gauge group $G$ ( $\operatorname{rank} G=r$ ) and matter chiral multiplets in representation $\otimes_{a=1}^{N_{\mathrm{F}}} R^{a}$. The saddle point equations in four dimensions are given by

$$
\begin{aligned}
\mathcal{D}_{\bar{z}} \phi_{a} & =0, \quad 2 i F_{z \bar{z}}^{\alpha}+g^{2}\left(\phi_{a}^{\dagger} T_{R^{a}}^{\alpha} \phi_{a}-r^{\alpha}\right)=0 \\
F_{\xi \bar{\xi}} & =F_{\xi z}=F_{\xi \bar{z}}=\mathcal{D}_{\xi} \phi_{a}=\mathcal{D}_{\xi} \bar{\phi}_{a}=0
\end{aligned}
$$

where $T_{R^{a}}^{\alpha}(\alpha=1, \cdots, \operatorname{dim} G)$ are the generators of the gauge group $G$ and $r^{\alpha}$ are FI parameters which are non-zero only for the central U(1) parts of the gauge group. As in the Abelian case, the first two equations can be identified with the BPS vortex equations in the trivial $2 \mathrm{~d}$ plane $[10-17]$ and the other equations restrict the vortex configurations to be at the fixed points of the isometry $\mathcal{Q}^{2}$ on the moduli space.

In the following, we restrict ourselves to the case where the saddle point configurations have no flat direction. Then we can show that each saddle point are specified by a set of flavor labels $\mathbf{a}=\left(a_{1}, a_{2}, \cdots, a_{r}\right)$, a linearly independent set of weight vectors $\overrightarrow{\boldsymbol{\mu}}=\left(\vec{\mu}_{1}, \vec{\mu}_{2}, \cdots, \vec{\mu}_{r}\right) \in\left(R^{a_{1}}, R^{a_{2}}, \cdots, R^{a_{r}}\right)$ and a set of non-negative integers $\mathbf{k}=\left(k_{1}, k_{2}, \cdots, k_{r}\right)$. The flavor labels a and the weight vectors $\overrightarrow{\boldsymbol{\mu}}$ specify the non-zero components of the scalar fields ${ }^{7} \phi_{a}$, whereas the integers $\mathbf{k}$ denote their winding numbers. In general, the saddle point configurations take the form

$$
A_{\bar{z}}=-\frac{i}{2} \vec{H} \cdot \partial_{\bar{z}} \vec{\omega}, \quad A_{\xi}=\vec{H} \cdot \vec{\sigma}_{*}, \quad \phi_{\rho}^{a}=\exp \left(-\frac{1}{2} \vec{\rho} \cdot \vec{\omega}\right) h_{\rho}^{a}(z),
$$

where $\vec{H}$ stands for the generators of the Cartan subalgebra $\mathfrak{t} \subset \mathfrak{g}$ and $\vec{\omega}=\left(\omega_{1}, \omega_{2}, \cdots, \omega_{r}\right)$ are profile functions, whose explicit forms are not important. Each component of the scalar fields $\phi_{\rho}^{a}\left(a=1, \cdots, N_{\mathrm{F}}, \vec{\rho} \in R^{a}\right)$ is determined by a holomorphic polynomial $h_{\rho}^{a}(z)$. For the saddle point specified by $\mathbf{a}, \overrightarrow{\boldsymbol{\mu}}$ and $\mathbf{k}$, the polynomials $h_{\rho}^{a}(z)$ are given by

$$
h_{\rho}^{a}(z)=\left\{\begin{array}{cl}
z^{k_{i}} & \left(a=a_{i}, \vec{\rho}=\vec{\mu}_{i}\right) \\
0 & \text { (otherwise) }
\end{array} .\right.
$$

\footnotetext{
${ }^{7}$ The $D$-term condition requires that if $a_{i}=a_{j}$, the difference of the corresponding weight vectors cannot be on the root lattice $\vec{\mu}_{i}-\vec{\mu}_{j} \notin \Delta_{G}$.
} 
The constants $8 \vec{\sigma}_{*}=\left(\sigma_{01}, \sigma_{02}, \cdots, \sigma_{0 r}\right)$ can be determined from eq. (2.86), which reduces to the following condition:

$$
\vec{\mu}_{i} \cdot \vec{\sigma}_{*}=-m_{a_{i}}-\epsilon k_{i} \quad(i=1, \cdots, r) .
$$

Since the weight vectors $\left(\vec{\mu}_{1}, \vec{\mu}_{2}, \cdots, \vec{\mu}_{r}\right)$ are linearly independent, this system of equations uniquely determine $\vec{\sigma}_{*}$.

As in the Abelian case, the computation of the one-loop determinants essentially reduces to the problem of finding solutions of the linearized BPS vortex equations. We can show that they are specified by a set of holomorphic functions $\delta h_{\rho}^{a}(z)$ :

$$
\delta \phi_{\rho}^{a}=e^{-\frac{1}{2} \vec{\rho} \cdot \vec{\omega}}\left[\delta h^{a}(z)+\delta \Omega \cdot h^{a}\right]_{\rho}, \quad \delta A_{\bar{z}}=e^{-\frac{1}{2} \vec{H} \cdot \vec{\omega}}\left(i \partial_{\bar{z}} \delta \Omega\right) e^{\frac{1}{2} \vec{H} \cdot \vec{\omega}},
$$

and $\delta A_{\xi}=0$. Here, $\delta \Omega(z, \bar{z})$ is a function which takes value in the complexified Lie algebra $\mathfrak{g}^{\mathbb{C}}$ and in principle, it can be determined once $\delta h_{\rho}^{a}(z)$ are given. In the $2 \mathrm{~d}$ limit, each term in the Taylor expansion $\delta h_{\rho}^{a}(z)=\sum c_{\rho, l}^{a} z^{l}$ has the eigenvalue $-i \mathcal{D}_{\xi} \doteq \vec{\rho} \cdot \vec{\sigma}_{*}+m_{a}+l \epsilon$, so that each component $\delta h_{\rho}^{a}$ gives the following contribution to $\operatorname{det}\left(-i \mathcal{D}_{\xi}\right)^{-1}$ :

$$
\left.\frac{1}{\operatorname{det}\left(-i \mathcal{D}_{\xi}\right)}\right|_{a, \rho}=\prod_{l=0}^{\infty} \frac{\Lambda_{0}}{\vec{\rho} \cdot \vec{\sigma}_{*}+m_{a}+l \epsilon}=\left(\frac{\Lambda_{0}}{\epsilon}\right)^{-\frac{\vec{\rho} \cdot \vec{\sigma}_{*}+m_{a}}{\epsilon}} \Gamma\left(\frac{\vec{\rho} \cdot \vec{\sigma}_{*}+m_{a}}{\epsilon}\right) .
$$

The total contribution can be obtained by taking the product over all the components $\delta h_{\rho}^{a}$ and eliminating the contributions from the unphysical modes generated by the infinitesimal complexified gauge transformation defined by

$$
\delta h^{a} \sim \delta h^{a}(z)+v(z) \cdot h^{a}(z), \quad \delta \Omega \sim \delta \Omega-v(z) .
$$

The transformation parameter $v(z)$ is an arbitrary element of the complexified Lie algebra $\mathfrak{g}^{\mathbb{C}}$, which can be decomposed into the diagonal and off-diagonal parts

$$
v(z)=\vec{P}(z) \cdot \vec{H}+\sum_{\vec{\alpha} \in \Delta_{G}} Q_{\vec{\alpha}}(z) E_{\vec{\alpha}}
$$

where $\vec{P}(z)$ and $Q_{\vec{\alpha}}(z)$ are arbitrary polynomials.

By using the Cartan part $\vec{P}(z) \cdot \vec{H}$, the fluctuations of the non-zero scalar fields can be fixed to finite order polynomials

$$
\delta h_{\rho}^{a}=\sum_{l=0}^{k_{i}-1} c_{\rho, l}^{a} z^{l} \quad\left(\text { for } a=a_{i}, \vec{\rho}=\vec{\mu}_{i}\right) .
$$

Therefore, the contributions from the unphysical modes generated by the diagonal part $v(z)=\vec{P}(z) \cdot \vec{H}$ can be eliminated by replacing the infinite products eq. (2.91) for $a=$ $a_{i}, \vec{\rho}=\vec{\mu}_{i}$ with the finite ones

$$
\prod_{l=0}^{k_{i}-1} \frac{\Lambda_{0}}{\vec{\mu}_{i} \cdot \vec{\sigma}_{*}+m_{a_{i}}+l \epsilon}=\left(-\frac{\Lambda_{0}}{\epsilon}\right)^{k_{i}} \frac{1}{k_{i} !},
$$

\footnotetext{
${ }^{8}$ In two dimensions, $\vec{\sigma}_{*}$ specify the eigenvalues of the vector multiplet scalar field $\sigma$ at $z=0$.
} 
where we have used eq. (2.89). On the other hand, the off-diagonal part of the form $v(z)=z^{l} E_{\vec{\alpha}}$ generates the following unphysical mode:

$$
\delta h_{a}=z^{l} E_{\vec{\alpha}} \cdot h_{a} .
$$

The eigenvalue of this unphysical fluctuation is $-i \mathcal{D}_{\xi} \doteq \vec{\alpha} \cdot \vec{\sigma}_{*}+l \epsilon$. Therefore, the total contribution from the off-diagonal part $\sum Q_{\vec{\alpha}}(z) E_{\vec{\alpha}}$ can be eliminated by dividing $\operatorname{det}\left(-i \mathcal{D}_{\xi}\right)^{-1}$ with $^{9}$

$$
\prod_{\alpha \in \Delta_{G}} \prod_{l=0}^{\infty} \frac{\Lambda_{0}}{\vec{\alpha} \cdot \vec{\sigma}_{*}+l \epsilon}=\prod_{\alpha \in \Delta_{G}} \Gamma\left(\frac{\vec{\alpha} \cdot \vec{\sigma}_{*}}{\epsilon}\right) .
$$

For the $\mathcal{Q}$-closed part $\mathcal{I}$, we consider an analogue of eq. (2.66), whose value at the saddle point is given by

$$
\left.\exp (\mathcal{I})\right|_{\text {saddle point }}=\exp \left(-\frac{2 \pi i \vec{\sigma}_{*} \cdot \vec{\tau}_{0}}{\epsilon}\right)
$$

Although the FI parameters and theta angle can be assigned only for each central U(1) factor in the gauge group $G$, we have introduced auxiliary parameters $\vec{\tau}_{0}=\left(\tau_{01}, \cdots, \tau_{0 r}\right)$ for all the $\mathrm{U}(1)$ factors in the Cartan subgroup for later convenience.

Combining eq. (2.98) with the functional determinant eq. (2.91) and taking into account the unphysical contributions eq. (2.95) and (2.97), we obtain the following contribution to the vortex partition function from the saddle point $(\mathbf{a}, \overrightarrow{\boldsymbol{\mu}}, \mathbf{k})$

$$
Z_{\mathbf{a}, \boldsymbol{\mu}, \mathbf{k}}=\exp \left(-\frac{2 \pi i \vec{\sigma}_{*} \cdot \vec{\tau}}{\epsilon}\right)\left[\prod_{\vec{\alpha} \in \Delta_{G}} \Gamma\left(\frac{\vec{\alpha} \cdot \vec{\sigma}_{*}}{\epsilon}\right)\right]^{-1}\left[\prod_{i=1}^{r} \frac{(-1)^{k_{i}}}{k_{i} !}\right] \prod_{a, \rho}^{\prime} \Gamma\left(\frac{\vec{\rho} \cdot \vec{\sigma}_{*}+m_{a}}{\epsilon}\right)
$$

where $\prod^{\prime}$ denotes the product over all the chiral multiplets excluding $(a, \vec{\rho})=\left(a_{i}, \vec{\mu}_{i}\right)(i=$ $1, \cdots, r)$. Note that the UV scale has been absorbed into the renormalized coupling constants $\vec{\tau}$

$$
\frac{2 \pi i \vec{\sigma}_{*} \cdot \vec{\tau}}{\epsilon}=\frac{2 \pi i \vec{\sigma}_{*} \cdot \vec{\tau}_{0}}{\epsilon}+\sum_{a=1}^{N_{\mathrm{F}}} \sum_{\vec{\rho} \in R_{a}} \frac{\vec{\rho} \cdot \vec{\sigma}_{*}}{\epsilon} \log \left(\frac{\Lambda_{0}}{\epsilon}\right) .
$$

The vortex partition function in the vacuum specified by $(\mathbf{a}, \overrightarrow{\boldsymbol{\mu}})$ can be obtained by summing up eq. (2.99) over the winding numbers: $Z_{\mathbf{a}, \boldsymbol{\mu}}=\sum_{\mathbf{k}} Z_{\mathbf{a}, \boldsymbol{\mu}, \mathbf{k}}$. One can easily see from eq. (2.99) that $Z_{\mathbf{a}, \mu}$ can be rewritten into the following integral form:

$$
Z_{\mathbf{a}, \boldsymbol{\mu}}=\int_{C_{\mathbf{a}, \boldsymbol{\mu}}} \prod_{i=1}^{r}\left(\frac{d \sigma_{i}}{2 \pi i \epsilon}\right) \exp \left(-\frac{2 \pi i \vec{\sigma} \cdot \vec{\tau}}{\epsilon}\right)\left[\prod_{\vec{\alpha} \in G} \Gamma\left(\frac{\vec{\alpha} \cdot \vec{\sigma}}{\epsilon}\right)\right]^{-1} \prod_{a=1}^{N_{\mathrm{F}}} \prod_{\vec{\rho} \in R_{a}} \Gamma\left(\frac{\vec{\rho} \cdot \vec{\sigma}+m_{a}}{\epsilon}\right),
$$

\footnotetext{
${ }^{9}$ By using the reflection formula for the gamma function, the unphysical contribution can also be written as

$$
\left[\prod_{\alpha \in \Delta_{G}} \Gamma\left(\frac{\vec{\alpha} \cdot \vec{\sigma}_{*}}{\epsilon}\right)\right]^{-1}=\prod_{\vec{\alpha}>0} \frac{\vec{\alpha} \cdot \vec{\sigma}_{*}}{\pi \epsilon} \sin \left(-\frac{\pi \vec{\alpha} \cdot \vec{\sigma}_{*}}{\epsilon}\right) .
$$
}

This is called the Sklyanin measure [35]. 
where $C_{\mathbf{a}, \boldsymbol{\mu}}$ is a contour which encloses the set of poles located at $\vec{\sigma}=\vec{\sigma}_{*}$ satisfying the saddle point condition $\overrightarrow{\boldsymbol{\mu}} \cdot \vec{\sigma}_{*}=-\mathbf{m}-\epsilon \mathbf{k}$ with $\mathbf{k}=\left(k_{1}, \cdots, k_{r}\right) \in \mathbb{Z}_{\geq 0}^{r}$.

Let us remark that the expression (2.101) is indeed equivalent to the hemisphere partition function formula [31-33] with the Neumann boundary condition applied to the chiral multiplets. This agreement is reasonable because the two-dimensional Euclidean space $\mathbf{R}^{2}$ is topologically equivalent to the hemisphere, where the infinity in this case plays a role of the boundary. From this point of view, it would be interesting to study a situation corresponding to the Dirichlet boundary condition. It shall be possible if we put physical degrees of freedom at infinity.

Fractional vortices. Here, we briefly see that the factorization of property of the vortex partition function eq. (2.78) is slightly modified when there exist the so-called fractional vortices [36]. For the set of weight vectors $\boldsymbol{\mu}=\left(\vec{\mu}_{1}, \cdots, \vec{\mu}_{r}\right)$, let $\left\{\vec{\mu}_{1}^{*}, \cdots, \vec{\mu}_{r}^{*}\right\}$ be the set of vectors such that

$$
\vec{\mu}_{i} \cdot \vec{\mu}_{j}^{*}=\delta_{i j} .
$$

Once the solution to the saddle point condition eq. (2.89) for $\mathbf{k}=0$ is given, those for other configurations can be written as

$$
\vec{\sigma}_{\mathbf{a}, \boldsymbol{\mu}, \mathbf{k}}=\vec{\sigma}_{\mathbf{a}, \boldsymbol{\mu}, \mathbf{0}}-\epsilon \sum_{i=1}^{r} k_{i} \vec{\mu}_{i}^{*}
$$

In other words, the difference $\vec{d}_{\mathbf{a}, \boldsymbol{\mu}, \mathbf{k}} \equiv \epsilon^{-1}\left(\vec{\sigma}_{\mathbf{a}, \boldsymbol{\mu}, \mathbf{k}}-\vec{\sigma}_{\mathbf{a}, \boldsymbol{\mu}, \mathbf{0}}\right)$ is on the lattice $\Lambda_{\boldsymbol{\mu}}^{*}$ generated by $\left\{\vec{\mu}_{1}^{*}, \cdots, \vec{\mu}_{r}^{*}\right\}$, which contains the cocharactor lattice $\Lambda_{\text {cochar }}$ as a sublattice

$$
\Lambda_{\mu}^{*} \equiv\left\{\vec{\lambda} \mid \vec{\lambda} \cdot \vec{\mu}_{i} \in \mathbb{Z}, 1 \leq i \leq r\right\} \supseteq \Lambda_{\text {cochar }} \equiv\left\{\vec{\lambda} \mid \vec{\lambda} \cdot \vec{\rho} \in \mathbb{Z}, \forall \vec{\rho} \in \cup_{a} R_{a}\right\} .
$$

If there is a weight $\vec{\rho} \in \cup_{a} R_{a}$ such that $\vec{\rho} \cdot \vec{\mu}_{i}^{*} \notin \mathbb{Z}$, the lattice $\Lambda_{\mu}^{*}$ contains $\Lambda_{\text {cochar }}$ as a proper subgroup, i.e. $\Lambda_{\mu}^{*} \neq \Lambda_{\text {cochar. }}$. In such a case, one can show that the classical vacuum specified by $\boldsymbol{\mu}$ has an unbroken discrete gauge symmetry. It has been known that in such a classical vacuum, there can be the so-called fractional vortices, whose magnetic fluxes are fractional numbers. For a saddle point with $\vec{d}_{\mathbf{a}, \mu, \mathbf{k}} \notin \Lambda_{\text {cochar }}$, the magnetic flux $\int F \propto \vec{H} \cdot \vec{d}_{\mathbf{a}, \boldsymbol{\mu}, \mathbf{k}}$ has fractional eigenvalues, and hence there exist fractional vortices in such a saddle point configuration.

In the previous section, we have seen in eq. (2.78) that the vortex partition function factorizes into the perturbative and vortex parts. However, this is not always the case when there exist fractional vortices. This is because the ratio $Z_{\mathbf{a}, \boldsymbol{\mu}, \mathbf{k}} / Z_{\mathbf{a}, \boldsymbol{\mu}, \mathbf{0}}$ is not necessarily a rational function which can be written as a finite dimensional integral over the vortex moduli space

$$
\frac{Z_{\mathbf{a}, \boldsymbol{\mu}, \mathbf{k}}}{Z_{\mathbf{a}, \boldsymbol{\mu}, \mathbf{0}}} \sim e^{2 \pi i k_{i} \vec{\mu}_{i}^{*} \cdot \vec{\tau}} \prod_{b, \rho} \frac{\Gamma\left(x_{b, \rho}-k_{i} \vec{\rho} \cdot \vec{\mu}_{i}^{*}\right)}{\Gamma\left(x_{b, \rho}\right)}, \quad x_{b, \rho} \equiv \frac{\vec{\rho} \cdot \vec{\sigma}_{\mathbf{a}, \boldsymbol{\mu}, \mathbf{0}}+m_{b}}{\epsilon} .
$$

In such a case, the vortex partition function decomposes into several sectors labeled by the quotient $\Lambda_{\boldsymbol{\mu}}^{*} / \Lambda_{\text {cochar }}$, each of which has a factorized form

$$
Z_{\mathbf{a}, \boldsymbol{\mu}}=\sum_{w} Z_{\mathbf{a}, \boldsymbol{\mu}, p}^{\mathrm{pert}} Z_{\mathbf{a}, \boldsymbol{\mu}, p}^{\mathrm{vortex}}, \quad w \in \Lambda_{\boldsymbol{\mu}}^{*} / \Lambda_{\text {cochar }} .
$$

We will see an example of fractional vortices in the next section. 


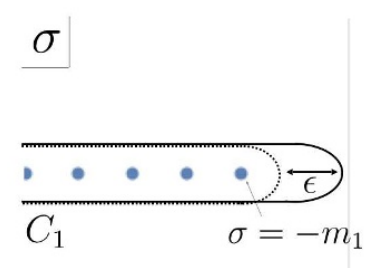

Figure 2. The vortex partition function $Z_{1}$ is invariant under the shift of the contour $C_{1}$.

Let us comment on another realization of fractional vortices, which appear in the two-dimensional orbifold $\mathbb{C} / \mathbb{Z}_{p}$ plane [37] for which the vortex counting was studied in ref. [38]. This fractional vortex is different from that mentioned here, because the current one corresponds to orbifolding the target space. Although these two are different, it would be interesting to study them with emphasis on the similarity.

\section{Differential equation for the vortex partition function}

\section{1 $\mathrm{U}(1)$ gauge theories with $N$ chiral multiplets}

In the previous section, we have obtained the integral expression for the vortex partition function. It is also convenient to characterize the vortex partition function in terms of linear differential equation which gives $Z_{a}(\tau)$ as certain linear combinations of its independent solutions.

Let us first start with the simplest example of U(1) gauge theory with $N$ charged chiral multiplets. The vortex partition function eq. (2.81) is invariant under an arbitrary deformation of the integration contour $C_{a}$ as long as it does not cross any pole of the integrand. Shifting the contour by $\epsilon$ and changing the integration variable $\sigma \rightarrow \sigma+\epsilon$, as shown in figure 2 , we obtain

$$
Z_{a}=\text { eq. }(2.81)=e^{-2 \pi i \tau} \int_{C_{a}} \frac{d \sigma}{2 \pi i \epsilon} \exp \left(-\frac{2 \pi i \sigma \tau}{\epsilon}\right) \prod_{b=1}^{N} \frac{\sigma+m_{a}}{\epsilon} \Gamma\left(\frac{\sigma+m_{b}}{\epsilon}\right) .
$$

This relation can be rewritten into the following linear differential equation:

$$
P(\hat{\sigma}) Z_{a} \equiv\left[\prod_{b=1}^{N}\left(\hat{\sigma}+m_{b}\right)-\epsilon^{N} e^{2 \pi i \tau}\right] Z_{a}=0
$$

where we have defined the differential operator $\hat{\sigma}$ by

$$
\hat{\sigma} \equiv-\frac{\epsilon}{2 \pi i} \partial_{\tau} .
$$


We can see that $Z_{a}(a=1, \cdots, N)$ in eq. (2.75) are the linearly independent solutions of this differential equation for which $\epsilon \log Z_{a}$ is analytic around $\epsilon=0$. This analyticity is an essential condition for the vortex partition function since $Z_{a}$ should have the following behavior for small $\epsilon$ :

$$
Z_{a}=\exp \left[-\frac{2 \pi}{\epsilon} \widetilde{W}_{a}+\mathcal{O}(1)\right]
$$

As we have seen in the previous section, $\widetilde{W}_{a}$ is the on-shell value of the twisted superpotential in the $a$-th vacuum, which is related to the VEV of $\sigma$ as

$$
\langle\sigma\rangle_{a}=-\frac{2 \pi}{\epsilon} \hat{\sigma} \widetilde{W}_{a}
$$

This implies that for small $\epsilon$, the operator $\hat{\sigma}$ is replaced by its expectation value

$$
P(\hat{\sigma}) Z_{a}=P\left(\langle\sigma\rangle_{a}\right) Z_{a}+\mathcal{O}(\epsilon)=0 .
$$

Therefore, the $\epsilon \rightarrow 0$ limit of the differential equation (3.2) implies that the expectation value of $\sigma$ in each vacuum agrees with one of the roots of the polynomial $P(\sigma)$ given by

$$
P(\sigma)=\prod_{a=1}^{N}\left(\sigma+m_{a}\right)-\Lambda^{N}
$$

Note that we have replaced the parameter $\tau(\epsilon)$ renormalized at $\mu=\epsilon$ with the $\mu$-independent scale $\Lambda$ defined by

$$
\Lambda=\mu \exp \left(\frac{2 \pi i \tau(\mu)}{N}\right), \quad \text { for arbitrary } \mu
$$

The off-shell twisted superpotential. Once the on-shell effective twisted superpotential $\widetilde{W}_{a}$ is obtained, the off-shell effective twisted superpotential $\widetilde{W}_{\text {eff }}(\tau, \sigma)$ can also be obtained by the so-called integrating-in procedure

$$
\widetilde{W}_{\mathrm{eff}}(\tau, \sigma)=i \sigma \tau(\mu)+\widetilde{W}_{L}(\sigma),
$$

where $\widetilde{W}_{L}(\sigma)$ is the Legendre transform of the on-shell twisted superpotential

$$
\widetilde{W}_{L}(\sigma)=\left.\left(\widetilde{W}_{\mathrm{on}}(\tau)-i \sigma \tau\right)\right|_{\tau=\tau(\sigma)}, \quad \tau(\sigma)=\frac{1}{2 \pi i} \log \prod_{a=1}^{N} \frac{\sigma+m_{a}}{\mu} .
$$

From the effective twisted superpotential $\widetilde{W}_{\text {eff }}(\tau, \sigma)$, the twisted $F$-term equation, which determines the expectation value of $\sigma$, can be obtained as

$$
1=\exp \left(2 \pi \partial_{\sigma} \widetilde{W}_{\text {eff }}\right)=e^{2 \pi i \tau(\mu)} \exp \left(2 \pi W_{L}^{\prime}(\sigma)\right) .
$$

On the other hand, we have seen that the expectation values of $\sigma$ satisfies $P(\sigma)=0$. Comparing these two equations, we conclude that

$$
\widetilde{W}_{\mathrm{eff}}(\tau, \sigma)=i \sigma \tau(\mu)-\frac{1}{2 \pi} \sum_{a=1}^{N}\left(\sigma+m_{a}\right)\left(\log \frac{\sigma+m_{a}}{\mu}-1\right) .
$$

up to an additive constant which does not depend on $\sigma$. This agrees with the known result [39-42]. 


\subsection{Generalization to non-Abelian gauge theories}

We have seen in the previous example that in the presence of the $\Omega$ deformation parameter $\epsilon$, the twisted $F$-term equation $P(\sigma)=0$ is promoted to the differential equation $P(\hat{\sigma}) Z=0$. In this sense, the differential equation $P(\sigma) Z=0$ can be regarded as a "quantized" version of the twisted F-term equation. The $\Omega$ deformation parameter $\epsilon$ plays the role of the "Planck constant" characterizing the commutation relation

$$
[\hat{\sigma}, \hat{\tau}]=i \frac{\epsilon}{2 \pi} .
$$

This also holds in the model with arbitrary gauge group and matter content. In general, there is a differential equation for each elements of the cocharacter lattice $\Lambda_{\text {cochar }}$, i.e. for each vector $\vec{\lambda}$ such that $\vec{\rho} \cdot \vec{\lambda} \in \mathbb{Z}, \forall \vec{\rho} \in \cup_{a} R_{a}$. For each $\vec{\lambda} \in \Lambda_{\text {cochar }}$, let $P_{\lambda}^{ \pm}(\vec{\sigma})$ be the coprime polynomials of $\vec{\sigma}=\left(\sigma_{1}, \cdots, \sigma_{r}\right)$ defined by

$$
\frac{P_{\lambda}^{+}(\vec{\sigma})}{P_{\lambda}^{-}(\vec{\sigma})}=\prod_{a=1}^{N_{\mathrm{F}}} \prod_{\rho \in R_{a}} \epsilon^{\vec{\rho} \cdot \vec{\lambda}}\left(\frac{\vec{\rho} \cdot \vec{\sigma}+m_{a}}{\epsilon}\right)_{\vec{\rho} \cdot \vec{\lambda}},
$$

where $(n)_{m} \equiv \Gamma(n+m) / \Gamma(n)$ denotes the Pochhammer symbol. From the invariance of eq. (2.101) under the shift of contour $C_{\mathbf{a}, \boldsymbol{\mu}}$ by $\epsilon \vec{\lambda}$, we find that the vortex partition function satisfies the following differential equation:

$$
\left[\Delta(\overrightarrow{\hat{\sigma}}+\epsilon \vec{\lambda}) P_{\lambda}^{+}(\overrightarrow{\hat{\sigma}})-\epsilon^{\vec{\rho}_{t} \cdot \vec{\lambda}} \exp \left\{2 \pi i \vec{\lambda} \cdot\left(\vec{\tau}+\vec{\rho}_{w}\right)\right\} \Delta(\overrightarrow{\hat{\sigma}}-\epsilon \vec{\lambda}) P_{\lambda}^{-}(\overrightarrow{\hat{\sigma}})\right] Z=0
$$

where we have defined

$$
\vec{\rho}_{t}=\sum_{a=1}^{N_{\mathrm{F}}} \sum_{\vec{\rho} \in R_{a}} \vec{\rho}, \quad \vec{\rho}_{w}=\frac{1}{2} \sum_{\vec{\alpha}>0} \vec{\alpha}, \quad \Delta(\vec{\sigma})=\prod_{\vec{\alpha}>0} \vec{\alpha} \cdot \vec{\sigma} .
$$

The vector $\overrightarrow{\hat{\sigma}}=\left(\hat{\sigma}_{1}, \cdots, \hat{\sigma}_{r}\right)$ denotes the differential operators defined by

$$
\hat{\sigma}_{i}=-\frac{\epsilon}{2 \pi i} \frac{\partial}{\partial \tau_{i}}
$$

Note that not all vectors $\vec{\lambda} \in \Lambda_{\text {cochar }}$ give independent differential equations but those for the basis $\vec{\lambda}_{i}(i=1, \cdots, r)$ are independent. In the limit $\epsilon \rightarrow 0$, the differential equation reduces to the following algebraic equation for $\vec{\sigma}$

$$
\prod_{a=1}^{N_{\mathrm{F}}} \prod_{\rho \in R_{a}}\left\{\vec{\rho} \cdot \vec{\sigma}+m_{a}\right\}^{\vec{\rho} \cdot \vec{\lambda}}=\mu^{\vec{\rho}_{t} \cdot \vec{\lambda}} \exp \left[2 \pi i \vec{\lambda} \cdot\left\{\vec{\tau}(\mu)+\vec{\rho}_{w}\right\}\right]
$$

where we have changed the renormalization scale from $\epsilon$ to a generic scale $\mu$ by using the following relation

$$
2 \pi i \vec{\tau}=2 \pi i \vec{\tau}(\mu)+\vec{\rho}_{t} \log \frac{\mu}{\epsilon}
$$


Eq. (3.18) can be viewed as the "classical limit" of the differential equation eq. (3.15) and as expected, it is the twisted $F$-term equation obtained from the following effective twisted superpotential:

$$
\widetilde{W}_{\mathrm{eff}}=2 \pi i \vec{\sigma} \cdot \vec{\tau}(\mu)+\sum_{\alpha} f\left(\frac{\vec{\alpha} \cdot \vec{\sigma}}{\mu}\right)-\sum_{a=1}^{N_{\mathrm{F}}} \sum_{\rho \in R_{a}} f\left(\frac{\vec{\rho} \cdot \vec{\sigma}+m_{a}}{\mu}\right) .
$$

where $f(x) \equiv x(\log x-1)$.

Fractional vortices. Here, we discuss an example of the fractional vortices in terms of the differential equation for $Z$. Let us consider the $\mathrm{U}(1)$ gauge theory coupled to two chiral multiplets with charge $\rho_{1}=1$ and $\rho_{2}=q \in \mathbb{Z}$. For $\lambda=1$, the differential equation is given by

$$
\left[\left(\hat{\sigma}+m_{1}\right) \prod_{j=0}^{q-1}\left(q \hat{\sigma}+m_{2}+j \epsilon\right)-\epsilon^{q+1} e^{2 \pi i \tau}\right] Z=0 .
$$

The general solution of this differential equation is the linear combination of the following hypergeometric functions

$$
\begin{aligned}
f_{1} & =e^{2 \pi i \frac{m_{1}}{\epsilon} \tau}{ }_{0} F_{q}\left(\left\{1+\frac{s-i}{q}\right\}_{0 \leq i \leq q-1} ;-\frac{e^{2 \pi i \tau}}{(-q)^{q}}\right), \\
f_{2, j} & =e^{2 \pi i \frac{m_{2}}{q \epsilon} \tau}{ }_{0} F_{q}\left(\left\{1+\frac{j-i}{q}\right\}_{i \neq j}, 1+\frac{j-s}{q} ;-\frac{e^{2 \pi i \tau}}{(-q)^{q}}\right) e^{2 \pi i \frac{j}{q} \tau},
\end{aligned}
$$

where $s=\frac{q m_{1}-m_{2}}{\epsilon}$. The coefficients of the linear combination for $Z$ in each vacuum should be chosen so that $\epsilon \partial_{\tau} \log Z$ is analytic around $\epsilon=0$. Two such linear combinations

$$
Z_{1}=\Gamma(-s) f_{1}, \quad Z_{2}=\sum_{j=0}^{q-1} \Gamma\left(\frac{s-j}{q}\right) f_{2, j},
$$

agree with the following integral expression of $Z$ along the contours $C_{1}$ and $C_{2}$ enclosing the poles at $\sigma=-m_{1}-\epsilon k$ and $\left(-m_{2}-\epsilon k\right) / q$ :

$$
Z_{a}=\int_{C_{a}} \frac{d \sigma}{2 \pi i} e^{-\frac{2 \pi i \sigma \tau}{\epsilon}} \Gamma\left(\frac{\sigma+m_{1}}{\epsilon}\right) \Gamma\left(\frac{q \sigma+m_{2}}{\epsilon}\right)
$$

Note that $Z_{2}$ consists of the $q$ sectors, each of which has the contribution $\exp (2 \pi i \tau j / q)$ from $j$ fractional vortices. Since the differential equation eq. (3.21) is invariant under the shift $\tau \rightarrow \tau+1$, other analytic solutions can be obtained by applying the shifts $\tau \rightarrow \tau+p$ ( $p=$ $1, \cdots, q-1)$ to $Z_{2}$

$$
Z_{2, p}=\sum_{j=0}^{q-1} e^{\frac{2 \pi i p}{q}} \Gamma\left(\frac{s-j}{q}\right) f_{2, j}
$$

As this examples shows, if there exist fractional vortices, the vortex partition function is not invariant under the shift of the theta angle $\theta \rightarrow \theta+2 \pi$. This is the consequence of the fractionality of the magnetic flux. 


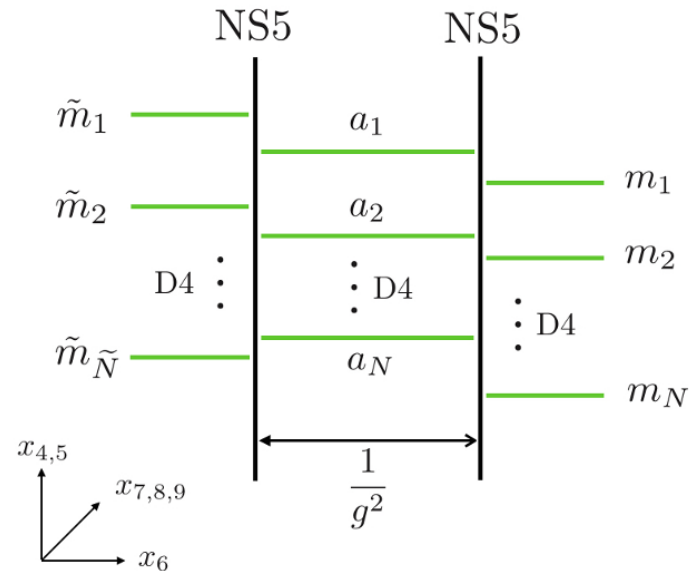

Figure 3. The Hanany-Witten brane setup.

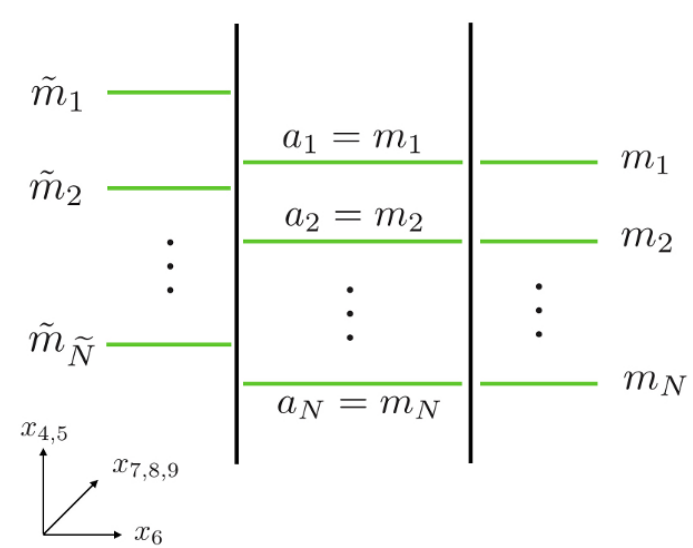

Figure 4. The root of the Higgs branch.

\section{Vortex counting on vortex string worldsheet}

In this section, we discuss vortex strings in four dimensions whose worldsheet effective dynamics is described by a certain $2 \mathrm{~d}$ model with $\mathcal{N}=(2,2)$ supersymmetry. It has been known that instanton-like vortices on the vortex string worldsheet can be identified with Yang-Mills instantons from the viewpoint of the parent 4d theory [29, 43, 44]. By using the differential equation discussed in the previous section, we show that the vortex partition functions in the $2 \mathrm{~d}$ vortex worldsheet theory agrees with the Nekrasov partition function at the so-called "root of the Higgs branch" in four dimensions.

The $4 \mathrm{~d}$ theory we consider is $\mathcal{N}=2 \mathrm{U}(N)$ gauge theory with $N$ fundamental and $\tilde{N}$ anti-fundamental hypermultiplets, which can be realized as the D4 branes worldvolume theory in the Hanany-Witten brane setup (figure 3). The root of the Higgs branch can be realized by connecting the D4 branes to the outer flavor branes (figure 4). This configuration corresponds to the point on the vacuum moduli space at which the Coulomb branch parameters $\vec{a}$ coincide with the masses of the fundamental hypermultiplets:

$$
\vec{a}=\vec{m} .
$$

The FI parameter $\xi_{4 d}$ can be introduced by shifting one of the NS5 branes in the $x^{7,8,9}$ direction. Then the theory is forced onto the Higgs branch, where there exist half BPS vortex strings corresponding to D2 branes stretched between the NS5 and D4 branes (figure 5). The worldsheet effective dynamics of $n$ vortex strings is described by $2 \mathrm{~d} \mathcal{N}=$ $(2,2) \mathrm{U}(n)$ gauge theory with one adjoint, $N$ fundamental and $\tilde{N}$ anti-fundamental chiral multiplets.

In the M-theory picture [45], the D4 and NS5 branes at a generic point on the Coulomb branch are identified with a single M5 brane described by the Seiberg-Witten curve. On the other hand, the M5 brane splits into two sheets at the root of Higgs branch [22], so that the Seiberg-Witten curve $F(v, t)$ takes the following factorized form:

$$
F(v, t)=P(v, t) Q(t),
$$




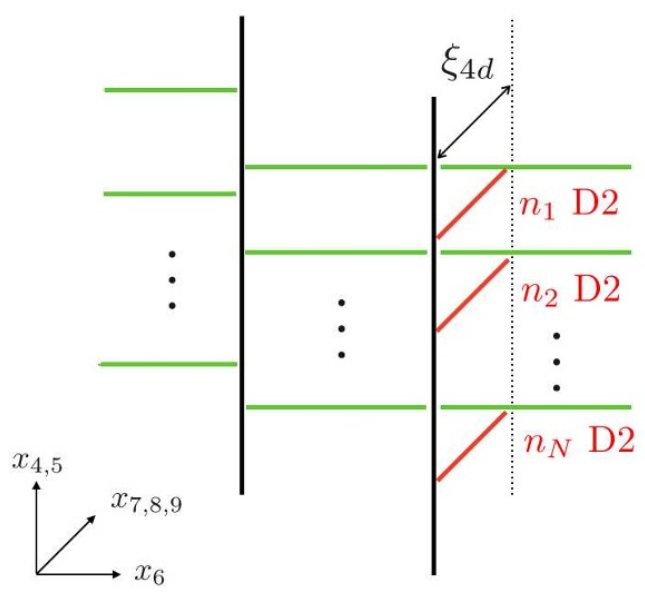

Figure 5. Vortex strings in the Higgs phase.

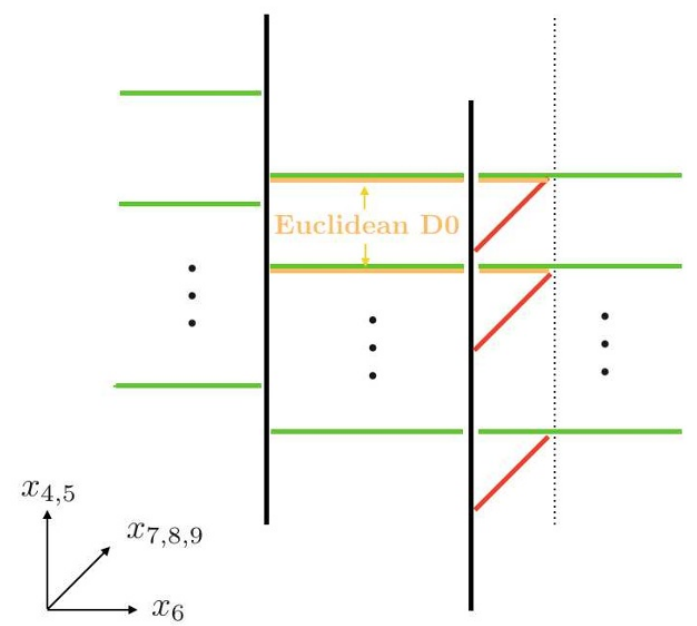

Figure 6. Instantons on vortex strings.

where the polynomials $P(v, t)$ and $Q(t)$ are given by

$$
P(v, t) \equiv \prod_{a=1}^{N}\left(v-m_{a}\right)-t \prod_{\tilde{a}=1}^{\tilde{N}}\left(v-\tilde{m}_{\tilde{a}}\right), \quad Q(t) \equiv t-\Lambda^{N-\tilde{N}} .
$$

One can regard $P(v, t)=0$ and $Q(t)=0$ as curves which determine how the two M5 branes are embedded in $\mathbb{R}^{3} \times S^{1}$ parameterized by $x_{4}+i x_{5} \propto v$ and $x_{6}+i x_{10} \propto \log \left(t / \Lambda^{N-\tilde{N}}\right)$.

The D2 branes, which corresponds to the BPS vortex strings, are lifted to M2 branes stretched between the M5 branes. Their positions $x_{4}+i x_{5} \propto-v_{i}(i=1, \cdots, n)$ can be determined from $P(v, t)=Q(t)=0$, that is,

$$
P\left(v_{i}, t=\Lambda^{N-\tilde{N}}\right)=\prod_{a=1}^{N}\left(v_{i}-m_{a}\right)-\Lambda^{N-\tilde{N}} \prod_{\tilde{a}=1}^{\tilde{N}}\left(v_{i}-\tilde{m}_{\tilde{a}}\right)=0 .
$$

In the $2 \mathrm{~d}$ vortex worldsheet theory, this equation appears as the effective twisted $F$-term equation which determines the eigenvalues of the adjoint scalar $\sigma_{i} \equiv-v_{i}$ in the $2 \mathrm{~d}$ vector multiplet.

It has been known that there is a correspondence between the BPS objects in two and four dimensions. At the root of the Higgs branch in four dimensions, there exist BPS monopoles (and dyons), which are confined and trapped by vortex strings. They are identified with BPS (dyonic) domain walls in the effective vortex worldsheet theory [46, 47]. Furthermore, we can show an exact agreement of the 2d/4d SUSY central charges for the BPS objects as a consequence of the close relationship between the Seiberg-Witten curve eq.(4.2) and the twisted $F$-term equations (4.4). This agreement implies that the nonperturbative corrections from instantons (Euclidean D0-branes in figure 6) are exactly identical in four and two dimensions.

In this section, we show the following equivalence between the $2 \mathrm{~d}$ vortex partition function and the $4 \mathrm{~d}$ instanton partition function:

$$
Z_{2 d, \vec{n}}\left(m_{a}, \tilde{m}_{\tilde{a}}, \epsilon_{1}, \epsilon_{2}, \tau\right)=\left.A(\tau) Z_{4 d}\left(a_{i}, m_{a}, \tilde{m}_{\tilde{a}}, \epsilon_{1}, \epsilon_{2}, \tau\right)\right|_{\vec{a}=\vec{m}-\epsilon_{1} \vec{n}},
$$


where $\vec{n}=\left(n_{1}, \cdots, n_{N}\right) \in \mathbb{Z}_{\geq 0}^{N}$ is a vector which specifies a vacuum in $2 \mathrm{~d}$ theory and $\mathrm{U}(1)^{N} \subset \mathrm{U}(N)$ magnetic fluxes of the vortex strings in $4 \mathrm{~d}$ theory (see below for details). $A(\tau)$ is a function which is independent of $\vec{n}$, so that it does not appear in the relation between the ratios of the partition functions for different $\vec{n}$

$$
\frac{Z_{2 d, \vec{n}}}{Z_{2 d, \vec{n}^{\prime}}}=\frac{Z_{4 d, \vec{a}=\vec{m}-\epsilon_{1} \vec{n}}}{Z_{4 d, \vec{a}=\vec{m}-\epsilon_{1} \vec{n}^{\prime}}}
$$

As a consequence of this relation, we can prove several known $2 \mathrm{~d} / 4 \mathrm{~d}$ relations. When $\epsilon_{2}$, the $\Omega$-deformation parameter along the vortex worldsheet, is turned off, the above relation reduces to

$$
\widetilde{\mathcal{W}}_{\vec{n}}-\widetilde{\mathcal{W}}_{\vec{n}^{\prime}}=\lim _{\epsilon_{2} \rightarrow 0}\left(-\frac{\epsilon_{2}}{2 \pi} \log \frac{Z_{4 d, \vec{a}=\vec{m}-\epsilon_{1} \vec{n}}}{Z_{4 d, \vec{a}=\vec{m}-\epsilon_{1} \vec{n}^{\prime}}}\right)
$$

where we have used the relation between the vortex partition function and the on-shell value of the superpotential

$$
Z_{2 d, \vec{n}}=\exp \left(-\frac{2 \pi}{\epsilon_{2}} \widetilde{\mathcal{W}}_{\vec{n}}+\mathcal{O}(1)\right)
$$

Eq. (4.7) indicates the correspondence between the $2 \mathrm{~d}$ domain wall central charge and the Nekrasov-Shatashvili limit of the instanton partition function [48, 49]. Furthermore, if both of the $\Omega$-deformation parameters $\left(\epsilon_{1}, \epsilon_{2}\right)$ are turned off, the relation reduces to that for the SUSY central charges for the $2 \mathrm{~d}$ domain walls between the vacua $\vec{n}$ and $\vec{n}^{\prime}$ and the $4 \mathrm{~d}$ monopoles with magnetic charge $\vec{n}-\vec{n}^{\prime}[21,22,29,30]$ :

$$
\lim _{\epsilon_{1} \rightarrow 0}\left(\widetilde{\mathcal{W}}_{\vec{n}}-\widetilde{\mathcal{W}}_{\vec{n}^{\prime}}\right)=-\frac{1}{2 \pi}\left(\vec{n}-\vec{n}^{\prime}\right) \cdot \frac{\partial}{\partial \vec{a}} \mathcal{F}
$$

where we have used the following relation between the Nekrasov partition function and the 4d prepotential $\mathcal{F}$

$$
Z_{4 d}=\exp \left(\frac{1}{\epsilon_{1} \epsilon_{2}} \mathcal{F}+\mathcal{O}(1)\right)
$$

Therefore, the equivalence of the partition functions eq. (4.5) is one of the most fundamental relations in this type of $2 \mathrm{~d} / 4 \mathrm{~d}$ correspondence. In the following, we show eq. (4.5) by using the differential equation discussed in the previous section.

\subsection{The vortex worldsheet theory: Hanany-Tong model}

Let us first discuss the $2 \mathrm{~d}$ side of the correspondence, i.e. the effective wolrdsheet theory of $n$ vortex strings. This model, called the Hanany-Tong model [10], is described by $2 \mathrm{~d} \mathcal{N}=(2,2) \mathrm{U}(n)$ gauge theory coupled to one adjoint, $N$ fundamental and $\tilde{N}$ antifundamental chiral multiplets. Their scalar components are denoted by $B$ ( $n$-by- $n$ matrix), $I$ ( $n$-by- $N$ matrix $)$ and $J(\tilde{N}$-by-n matrix $)$, respectively. The gauge coupling constant in four dimensions is identified with the FI parameter in two dimensions and they are combined with the $\theta$-angles

$$
\tau=\frac{\theta_{2 \mathrm{~d}}}{2 \pi}+i r_{2 \mathrm{~d}}=\frac{\theta_{4 \mathrm{~d}}}{2 \pi}+i \frac{4 \pi}{g_{4 d}^{2}}
$$


One of the $4 \mathrm{~d} \Omega$-deformation parameters $\epsilon_{1}$ corresponds to the twisted mass for the adjoint chiral multiplet and the other parameter $\epsilon_{2}$ plays the role of $\Omega$-deformation parameter in two dimensions.

The conditions for the supersymmetric vacua are given by

$$
\begin{aligned}
{\left[B, B^{\dagger}\right]+I I^{\dagger}-J^{\dagger} J } & =\frac{4 \pi}{g_{4 d}^{2}} . \\
{[\sigma, B]=\epsilon_{1} B, \quad \sigma I+I M } & =0, \quad J \sigma+\left(\tilde{M}-\epsilon_{2}\right) J=0,
\end{aligned}
$$

where $M=\operatorname{diag}\left(m_{1}, \cdots, m_{N}\right)$ and $\tilde{M}-\epsilon_{2}=\operatorname{diag}\left(\tilde{m}_{1}-\epsilon_{2}, \cdots, \tilde{m}_{\tilde{N}}-\epsilon_{2}\right)$ are the twisted mass matrices for the chiral multiplets and the anti-chiral multiplets, respectively. The classical vacua of this model correspond to the BPS configurations of $n$ vortex strings in the $4 \mathrm{~d} \Omega$-background. For the Abelian vortices $(N=1)$, there exist unique vacuum, in which the scalar expectation values are given by

$$
B \propto\left(\begin{array}{cccc}
0 & 1 & & \\
& \ddots & \ddots & \\
& & 0 & 1 \\
& & & 0
\end{array}\right), \quad I \propto\left(\begin{array}{c}
0 \\
\vdots \\
0 \\
1
\end{array}\right)
$$

and $J=0$. For general $N$, vacuum configurations can be obtained by decomposing the matrices into $N$ blocks and embedding the above solution. The sizes $n_{a}(a=1, \cdots, N)$ of the diagonal blocks of $B$ are arbitrary as long as the total size is $n$. Thus, the vacua are labeled by a set of non-negative integers $\vec{n}=\left(n_{1}, \cdots, n_{N}\right)$ such that $\sum_{a=1}^{N} n_{a}=n$.

The saddle point configurations in this model are BPS vortex configurations corresponding to 4d Yang-Mills instantons trapped on the vortex worldsheets. In each vacuum, the saddle point condition eq. (2.89) for the classical eigenvalues of the vector multiplets scalar $\sigma$ takes the form

$$
\sigma_{a}^{q}-\sigma_{a}^{q+1}=\epsilon_{1}-k_{a}^{q} \epsilon_{2}, \quad \sigma_{a}^{n_{a}}=-m_{a}-k_{a}^{n_{a}} \epsilon_{2}, \quad\left(q=1, \cdots, n_{a}, a=1, \cdots, N\right),
$$

where we have decomposed the label of the eigenvalues $\sigma_{i}(i=1, \cdots, n)$ as $\sigma_{a}^{q}$. Solving this condition with respect to $\sigma_{a}^{q}$, we find that

$$
\sigma_{a}^{q}=-m_{a}+\left(n_{a}-q\right) \epsilon_{1}-\lambda_{a}^{q} \epsilon_{2}, \quad\left(\lambda_{a}^{q}-\lambda_{a}^{q+1}=k_{a}^{q}, \lambda_{a}^{n_{a}+1}=0\right) .
$$

Since the winding numbers $k_{a}^{q}$ are all non-negative, the integers $\lambda_{a}^{q}$ satisfy

$$
\lambda_{a}^{1} \geq \lambda_{a}^{2} \geq \cdots \geq \lambda_{a}^{n_{a}} \geq \lambda_{a}^{n_{a}+1}=0 .
$$

In other words, the saddle point vortex configurations in the vacuum $\vec{n}$ are labeled by a set of $N$ Young tableaux $Y_{a}=\left(\lambda_{a}^{1}, \lambda_{a}^{2}, \cdots\right)$ which have at most $n_{a}$ rows (since $\lambda_{a}^{q}=0$ for $\left.q \geq n_{a}+1\right)$.

The general formula eq. (2.101) implies that the vortex partition function in the vacuum $\vec{n}$ is given by the following integral along a path enclosing all the poles at eq. (4.16):

$$
Z_{2 d, \vec{n}}\left(\tau_{1}, \cdots, \tau_{n}\right)=\int_{C_{\vec{n}}} \prod_{i=1}^{n}\left[\frac{d \sigma_{i}}{2 \pi i \epsilon_{2}} \exp \left(-\frac{2 \pi i \sigma_{i} \tau_{i}}{\epsilon_{2}}\right)\right] Z_{g} Z_{a d} Z_{f} Z_{a f}
$$


where $Z_{g}, Z_{a d}, Z_{f}$ and $Z_{a f}$ are the contributions from the gauge, adjoint, fundamental and anti-fundamental degrees of freedom

$$
\begin{aligned}
& Z_{g}=\prod_{1 \leq i \neq j \leq n} \Gamma\left(\frac{\sigma_{i}-\sigma_{j}}{\epsilon_{2}}\right)^{-1}, \quad Z_{a d}=\prod_{i=1}^{n} \prod_{j=1}^{n} \Gamma\left(\frac{\sigma_{i}-\sigma_{j}-\epsilon_{1}}{\epsilon_{2}}\right), \\
& Z_{f}=\prod_{i=1}^{n} \prod_{a=1}^{N} \Gamma\left(\frac{\sigma_{i}+m_{a}}{\epsilon_{2}}\right), \quad Z_{a f}=\prod_{i=1}^{n} \prod_{\tilde{a}=1}^{\tilde{N}} \Gamma\left(-\frac{\sigma_{i}+\tilde{m}_{\tilde{a}}-\epsilon_{2}}{\epsilon_{2}}\right) .
\end{aligned}
$$

Since there is no fractional vortex in this model, the vortex partition function simply factorizes into perturbative and vortex parts

$$
Z_{2 d, \vec{n}}=Z_{2 d, \vec{n}}^{\mathrm{pert}} Z_{2 d, \vec{n}}^{\mathrm{vortex}}
$$

The perturbative part is given by the residue of the integrand in eq. (4.18) at the pole corresponding to the vacuum configuration

$$
Z_{2 d, \vec{n}}^{\mathrm{pert}}=\prod_{a=1}^{N} \prod_{q=1}^{n_{a}}\left[\exp \left(-\frac{2 \pi i \tau_{a}^{q} \underline{\sigma}_{a}^{q}}{\epsilon_{2}}\right) \prod_{b=1}^{N} \Gamma\left(\frac{\underline{\sigma}_{a}^{q}+m_{b}-n_{b} \epsilon_{1}}{\epsilon_{2}}\right) \prod_{\tilde{a}=1}^{\widetilde{N}} \Gamma\left(-\frac{\underline{\sigma}_{a}^{q}+\tilde{m}_{\tilde{a}}-\epsilon_{2}}{\epsilon_{2}}\right)\right]
$$

where $\underline{\sigma}_{a}^{q}$ are the eigenvalues in the classical vacuum configuration

$$
\underline{\sigma}_{a}^{q}=-m_{a}-\left(q-n_{a}\right) \epsilon_{1} .
$$

On the other hand, the vortex part is the collection of the contributions from all the saddle points, which can be written as a sum over the Young tableaux

$$
Z_{2 d, \vec{n}}^{\operatorname{vortex}}\left(\tau_{1}, \cdots, \tau_{n}\right)=\sum_{\vec{Y}} \exp \left(\sum_{a=1}^{N} \sum_{q=1}^{n_{a}} 2 \pi i \lambda_{a}^{q} \tau_{a}^{q}\right) Z_{2 d, \vec{n}}^{\vec{Y}}
$$

In order to compare this quantity with the Nekrasov partition function in four dimensions, it is convenient to use the differential equations discussed in the previous section

$$
P_{i}\left(\hat{\sigma}_{1}, \cdots, \hat{\sigma}_{n}\right) Z_{2 d, \vec{n}}\left(\tau_{1}, \cdots, \tau_{n}\right)=0, \quad(i=1, \cdots, n),
$$

where $\hat{\sigma}_{i}$ are the differential operator

$$
\hat{\sigma}_{i} \equiv-\frac{\epsilon_{2}}{2 \pi i} \frac{\partial}{\partial \tau_{i}}
$$

The explicit forms of the polynomials $P_{i}\left(\hat{\sigma}_{i}\right)$ can be obtained from the invariance of the integral eq. (4.18) under the shift of the integration path $\sigma_{i} \rightarrow \sigma_{i}+1$ :

$$
P_{i}=Q_{i}^{+}-\epsilon_{2}^{N-\tilde{N}} \exp \left(2 \pi i \tau_{i}\right) Q_{i}^{-},
$$


where $Q_{i}^{ \pm}(i=1, \cdots, n)$ are the following polynomials

$$
\begin{aligned}
& Q_{i}^{+}\left(\hat{\sigma}_{1}, \cdots, \hat{\sigma}_{n}\right)=\left[\prod_{j \neq i}\left(\hat{\sigma}_{i}-\hat{\sigma}_{j}-\epsilon_{1}\right)\left(\hat{\sigma}_{i}-\hat{\sigma}_{j}+\epsilon_{2}\right)\right]\left[\prod_{a=1}^{N}\left(\hat{\sigma}_{i}+m_{a}\right)\right], \\
& Q_{i}^{-}\left(\hat{\sigma}_{1}, \cdots, \hat{\sigma}_{n}\right)=\left[\prod_{j \neq i}\left(\hat{\sigma}_{i}-\hat{\sigma}_{j}+\epsilon_{1}\right)\left(\hat{\sigma}_{i}-\hat{\sigma}_{j}-\epsilon_{2}\right)\right]\left[\prod_{\tilde{a}=1}^{\tilde{N}}\left(\epsilon_{2}-\hat{\sigma}_{i}-\tilde{m}_{\tilde{a}}\right)\right] .
\end{aligned}
$$

Once the perturbative part eq. (4.22) is given, the differential equations can be rewritten in terms of the vortex part:

$$
P_{i}\left(\hat{\sigma}_{i}-\underline{\sigma}_{i}\right) Z_{2 d, \vec{n}}^{\mathrm{vortex}}=0,
$$

where the constants $\underline{\sigma}_{i}$ denote the classical VEVs eq. (4.23). This differential equation completely determine the vortex part $Z_{2 d, \vec{n}}^{\text {vortex }}$ since all the coefficients $Z_{2 d, \vec{n}}^{\vec{Y}}$ in the expansion eq. (4.24) can be uniquely determined by setting the first term $Z_{2 d, \vec{n}}^{\vec{Y}=\emptyset}=1$ and recursively solving eq. (4.30) order by order. We will use this fact to prove the equivalence between the Nekrasov partition function and the vortex partition function.

Let us remark that in the limit $\epsilon_{2} \rightarrow 0$, the differential equations eq. (4.25) for $\tilde{N}=N$ reduces to

$$
\prod_{j \neq i} \frac{\left(\sigma_{i}-\sigma_{j}-\epsilon_{1}\right)}{\left(\sigma_{i}-\sigma_{j}+\epsilon_{1}\right)}=(-1)^{N} e^{2 \pi i \tau} \frac{\prod_{\tilde{a}=1}^{N}\left(\sigma_{i}+\tilde{m}_{\tilde{a}}\right)}{\prod_{a=1}^{N}\left(\sigma_{i}+m_{a}\right)} .
$$

This is the twisted F-term equation of the Hanany-Tong model and as pointed out in $[48,49]$, this agrees with the Bethe ansatz equation of the quantum $\operatorname{SL}(2, \mathbb{R})$ spin chain, in which $\epsilon_{1}$ plays a role of the Planck constant. See also [18-20]. On the other hand, as we have seen in the previous section, $\epsilon_{2}$ is another Planck constant characterizing the commutation relation eq. (3.13). In this sense, the system of the differential equations (4.25) has two Planck constants associated with the two different quantization conditions. If both the Planck constants are turned off, the differential equations (4.25) reduce to the twisted $F$-term equations (4.4) which describe the M2-branes. In this sense, the differential equations (4.25) can be viewed as a "doubly quantized" version of the twisted $F$-term equations.

\subsection{Comparison with Nekrasov partition function}

Perturbative part. Let us first compare the perturbative part of the vortex partition function eq. (4.22) and that of the Nekrasov partition function

$$
\begin{aligned}
Z_{4 d}^{\text {pert }}= & \exp \left(-\frac{\vec{a} \cdot \vec{a}}{\epsilon_{1} \epsilon_{2}} \pi i \tau_{0}\right) \prod_{1 \leq a \neq b \leq N} \Gamma_{2}\left(a_{b}-a_{a} \mid \epsilon_{1}, \epsilon_{2}\right)^{-1} \\
& \times \prod_{b=1}^{N}\left[\prod_{a=1}^{N} \Gamma_{2}\left(a_{b}-m_{a} \mid \epsilon_{1}, \epsilon_{2}\right) \prod_{\tilde{a}=1}^{\tilde{N}} \Gamma_{2}\left(a_{b}+\epsilon_{+}-\tilde{m}_{\tilde{a}} \mid \epsilon_{1}, \epsilon_{2}\right)\right] .
\end{aligned}
$$


where $\epsilon_{+} \equiv \epsilon_{1}+\epsilon_{2}$ and $\Gamma_{2}\left(x \mid \epsilon_{1}, \epsilon_{2}\right)$ is the double gamma function

$$
\Gamma_{2}\left(x \mid \epsilon_{1}, \epsilon_{2}\right)=\prod_{n=0}^{\infty} \prod_{m=0}^{\infty} \frac{\Lambda_{0}}{x+n \epsilon_{1}+m \epsilon_{2}} .
$$

This factor is obtained using the double zeta function in the same way as (2.58).

Now let us focus on the root of Higgs branch. In the presence of vortex strings in the $\Omega$-background, the Coulomb branch parameters $\vec{a}$ are shifted from those in the undeformed theory eq. (4.1) as ${ }^{10}$

$$
\vec{a}=\vec{m}-\epsilon_{1} \vec{n} .
$$

Plugging this relation into eq. (4.32), we can show that the classical part, the vectorfundamental part, and the anti-fundamental part are respectively given by

$$
\begin{aligned}
& \exp \left(-\frac{\vec{a} \cdot \vec{a}}{\epsilon_{1} \epsilon_{2}} \pi i \tau_{0}\right)=\exp \left[-\frac{2 \pi i \tau_{0}}{\epsilon_{2}} \sum_{a=1}^{N} \sum_{q=1}^{n_{a}} \underline{\sigma}_{a}^{q}\right] e^{-\frac{\pi i \tau_{0}}{\epsilon_{1} \epsilon_{2}}\left(\vec{m} \cdot \vec{m}-n \epsilon_{1}^{2}\right)}, \\
& \frac{\Gamma_{2}\left(a_{b}-m_{a} \mid \epsilon_{1}, \epsilon_{2}\right)}{\Gamma_{2}\left(a_{b}-a_{a} \mid \epsilon_{1}, \epsilon_{2}\right)}=\prod_{q=1}^{n_{a}} \Gamma_{1}\left(\underline{\sigma}_{a}^{q}+m_{b}-n_{b} \epsilon_{1} \mid \epsilon_{2}\right), \\
& \Gamma_{2}\left(a_{b}+\epsilon_{+}-\tilde{m}_{\tilde{a}} \mid \epsilon_{1}, \epsilon_{2}\right)=\left[\prod_{q=1}^{n_{b}} \Gamma_{1}\left(\epsilon_{2}-\underline{\sigma}_{b}^{q}-\tilde{m}_{\tilde{a}} \mid \epsilon_{2}\right)\right] \Gamma_{2}\left(\epsilon_{+}+m_{b}-\tilde{m}_{\tilde{a}} \mid \epsilon_{1}, \epsilon_{2}\right),
\end{aligned}
$$

where we have used the relations between the Gamma functions

$$
\Gamma_{2}\left(x \mid \epsilon_{1}, \epsilon_{2}\right)=\Gamma_{1}\left(x \mid \epsilon_{2}\right) \Gamma_{2}\left(x+\epsilon_{1} \mid \epsilon_{1}, \epsilon_{2}\right), \quad \Gamma_{1}\left(x \mid \epsilon_{2}\right) \equiv \frac{1}{\sqrt{2 \pi}}\left(\frac{\Lambda_{0}}{\epsilon_{2}}\right)^{-\frac{x}{\epsilon_{2}}+\frac{1}{2}} \Gamma\left(\frac{x}{\epsilon_{2}}\right) .
$$

For the cancellation of the vector-fundamental part (4.36), in particular, for $a=b$, the diagonal factors of the vector multiplet contribution are needed in the expression (4.32). Since such factors are independent of the Coulomb moduli and mass parameters, we put them as a vacuum-independent factor.

Combining these three factors, we find that the perturbative part in four dimensions agrees with that in two dimensions eq. (4.22) up to an $\vec{n}$ independent factor:

$$
Z_{2 d, \vec{n}}^{\text {pert }}\left(\tau_{a}^{p}=\tau\right)=\left.A(\tau) Z_{4 d}^{\text {pert }}(\tau)\right|_{\vec{a}=\vec{m}-\epsilon_{1} \vec{n}} \cdot
$$

where the renormalized $\tau$-parameter is given by

$$
\tau=\tau_{0}+\frac{N-\tilde{N}}{2 \pi i} \log \frac{\Lambda_{0}}{\epsilon_{2}} .
$$

\footnotetext{
${ }^{10} \mathrm{~A}$ similar phenomenon can also be seen for the vortices in the $2 \mathrm{~d} \Omega$-background discussed in section 2 :$$
\left.\sigma\right|_{z=0}=-m_{a}-k \epsilon
$$ 
Instanton part. At the root of the Higgs branch $\vec{a}=\vec{m}-\epsilon_{1} \vec{n}$, the instanton part $Z_{4 d}^{\text {inst }}(\tau)$ can be promoted to the $n$-variable function defined by

$$
Z_{4 d}^{\text {inst }}\left(\tau_{1}, \cdots, \tau_{n}\right) \equiv \sum_{\vec{Y}} \exp \left(\sum_{a=1}^{N} \sum_{q=1}^{n_{a}} 2 \pi i \lambda_{a}^{q} \tau_{a}^{q}\right) Z_{4 d}^{\vec{Y}}
$$

We show that $Z_{4 d}^{\text {inst }}\left(\tau_{1}, \cdots, \tau_{n}\right)$ satisfies the differential equation (4.30) which gives the vortex part of the $2 \mathrm{~d}$ partition function.

The contribution from each instanton configuration is given by the following integral formula

$$
\begin{aligned}
Z_{4 d}^{\vec{Y}}= & \int \prod_{\alpha=1}^{|\vec{Y}|}\left[\frac{d \Phi_{\alpha}}{2 \pi i} \frac{\epsilon_{1}+\epsilon_{2}}{\epsilon_{1} \epsilon_{2}} \prod_{\beta<\alpha} \frac{\left(\Phi_{\alpha}-\Phi_{\beta}\right)^{2}\left\{\left(\Phi_{\alpha}-\Phi_{\beta}\right)^{2}-\left(\epsilon_{1}+\epsilon_{2}\right)^{2}\right\}}{\left\{\left(\Phi_{\alpha}-\Phi_{\beta}\right)^{2}-\epsilon_{1}^{2}\right\}\left\{\left(\Phi_{\alpha}-\Phi_{\beta}\right)^{2}-\epsilon_{2}^{2}\right\}}\right. \\
& \left.\times \prod_{b=1}^{N} \frac{1}{\left(\Phi_{\alpha}+a_{b}\right)\left(\epsilon_{1}+\epsilon_{2}-a_{b}-\Phi_{\alpha}\right)} \prod_{a=1}^{N}\left(-m_{a}-\Phi_{\alpha}\right) \prod_{\tilde{a}=1}^{\tilde{N}}\left(\epsilon_{1}+\epsilon_{2}-\tilde{m}_{\tilde{a}}-\Phi_{\alpha}\right)\right],
\end{aligned}
$$

where the integration contours for $\Phi_{\alpha}(\alpha=1, \cdots,|\vec{Y}|)$ are those which enclose the following poles specified by the Young tableaux $\vec{Y}$ :

$$
\Phi_{b}^{(p, q)}=-a_{b}-(q-1) \epsilon_{1}-(p-1) \epsilon_{2},
$$

where we have relabeled $\Phi_{\alpha}$ as $\Phi_{b}^{(p, q)}\left(b=1, \cdots, N,(p, q) \in Y_{b}\right)$.

The differential equation for $Z_{4 d}^{\text {inst }}\left(\tau_{1}, \cdots, \tau_{n}\right)$ can be obtained from a recursion relation for $Z_{4 d}^{\vec{Y}}$, which can be derived in the following way. Let $\vec{Y}^{\prime}$ be the Young tableaux obtained by adding a box to $\vec{Y}$. The integral representation for $Z_{4 d}^{\vec{Y}^{\prime}}$ has one more integration variable than that for $Z_{4 d}^{\vec{Y}}$. The contour for the new integration variable $\Phi$ should be chosen so that it encloses the pole corresponding to the added box, while for the other variable, the contours are kept the same as those for $Z_{4 d}^{\vec{Y}}$. Thus, $Z_{4 d}^{\vec{Y}}$ is contained in $Z_{4 d}^{\vec{Y}^{\prime}}$ as its $\Phi$-independent part and their ratio takes the form

$$
\begin{aligned}
\frac{Z_{4 d}^{\vec{Y}^{\prime}}}{Z_{4 d}^{\vec{Y}}}= & \int \frac{d \Phi}{2 \pi i} \frac{\epsilon_{1}+\epsilon_{2}}{\epsilon_{1} \epsilon_{2}}\left[\prod_{b=1}^{N} \prod_{p, q=0}^{\infty}\left\{\Phi+a_{b}+(q-1) \epsilon_{1}+(p-1) \epsilon_{2}\right\}^{I_{b}(p, q)}\right. \\
& \left.\times \prod_{b=1}^{N} \frac{1}{\left(\Phi+a_{b}\right)\left(\epsilon_{1}+\epsilon_{2}-a_{b}-\Phi\right)} \prod_{a=1}^{N}\left(-m_{a}-\Phi\right) \prod_{\tilde{a}=1}^{\tilde{N}}\left(\epsilon_{1}+\epsilon_{2}-\tilde{m}_{\tilde{a}}-\Phi\right)\right] .
\end{aligned}
$$

The exponents $I_{b}(p, q)$ can be determined from the Young tableau $Y_{b}$ as (see figure 7)

$$
I_{b}(p, q)=\left[2 \rho_{b}^{(p, q)}+\rho_{b}^{(p+1, q+1)}+\rho_{b}^{(p-1, q-1)}\right]-\left[\rho_{b}^{(p-1, q)}+\rho_{b}^{(p+1, q)}+\rho_{b}^{(p, q+1)}+\rho_{b}^{(p, q-1)}\right],
$$

where $\rho_{b}^{(p, q)}$ is one or zero depending on if $(p, q) \in Y_{b}$ or $(p, q) \notin Y_{b}$. Eq. (4.44) can be further simplified by using the relation

$$
\prod_{p=0}^{\infty}\left\{\Phi+a_{b}+(q-1) \epsilon_{1}+(p-1) \epsilon_{2}\right\}^{I_{b}(p, q)}=\frac{\left(\Phi-\Phi_{b}^{q}\right)\left(\Phi-\Phi_{b}^{q-1}+\epsilon_{1}+\epsilon_{2}\right)}{\left(\Phi-\Phi_{b}^{q+1}-\epsilon_{1}\right)\left(\Phi-\Phi_{b}^{q}+\epsilon_{2}\right)},
$$




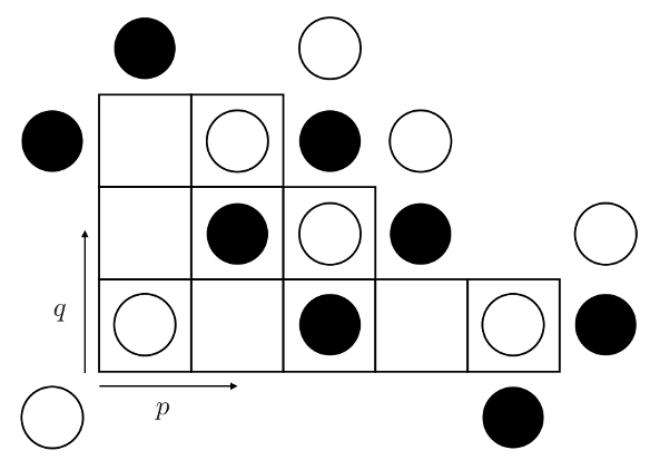

Figure 7. The exponents $I_{b}(p, q)$ for a tableau: $I_{b}(p, q)=1$ and $I_{b}(p, q)=-1$ at the points with the white and black circles, respectively.

where $\Phi_{b}^{q}$ is given by

$$
\Phi_{b}^{q}=-a_{b}-(q-1) \epsilon_{1}-\left(\lambda_{b}^{q}-1\right) \epsilon_{2}, \quad\left(\lambda_{b}^{q}=0 \text { for } q=0,-1\right) .
$$

Now let us focus on the root of the Higgs branch. When $a_{b}=m_{b}-\epsilon_{1} n_{b}$, the pole corresponding to the box at $\left(1, n_{b}+1\right)$ disappears. Thus, all the contributions from $Y_{b}$ with more than $n_{b}$ rows vanish and the infinite product over $q$ in eq. (4.44) terminates at $q=n_{b}+1$ for $Y_{b}$ with $\lambda_{b}^{n_{b}+1}=0$. Integrating eq. (4.44) around the pole at $\Phi=\Phi_{b}^{p}-\epsilon_{2}$, we find taht

$$
\frac{Z_{\vec{Y}^{\prime}}}{Z_{\vec{Y}}}=(-1)^{\tilde{N}} \prod_{(a, q) \neq(b, p)} \frac{\left(\Phi_{b}^{p}-\Phi_{a}^{q}-\epsilon_{2}\right)\left(\Phi_{b}^{p}-\Phi_{a}^{q}+\epsilon_{1}\right)}{\left(\Phi_{b}^{p}-\Phi_{a}^{q}-\epsilon_{1}-\epsilon_{2}\right)\left(\Phi_{b}^{p}-\Phi_{a}^{q}\right)} \frac{\prod_{\tilde{a}=1}^{\tilde{N}}\left(\Phi_{b}^{p}+\tilde{m}_{\tilde{a}}-\epsilon_{1}-2 \epsilon_{2}\right)}{\prod_{a=1}^{N}\left(\Phi_{b}^{p}+m_{a}-\epsilon_{1}-2 \epsilon_{2}\right)} .
$$

This is the recursion relation which can be obtained by substituting

$$
Z_{4 d}^{\text {inst }}\left(\tau_{1}, \cdots, \tau_{n}\right) \equiv \sum_{\vec{Y}} \exp \left(\sum_{a=1}^{N} \sum_{q=1}^{n_{a}} 2 \pi i \lambda_{a}^{q} \tau_{a}^{q}\right) Z_{4 d}^{\vec{Y}}
$$

into the differential equation eq. (4.30). Therefore, $Z_{4 d}^{\text {inst }}\left(\tau_{1}, \cdots, \tau_{n}\right)$ satisfies the differential equation which uniquely determines the vortex part of the $2 \mathrm{~d}$ partition function, and hence the non-perturbative parts of the $2 \mathrm{~d}$ and $4 \mathrm{~d}$ partition functions are identical to each other

$$
Z_{2 d, \vec{n}}^{\text {vortex }}\left(\tau_{1}, \cdots, \tau_{n}\right)=\left.Z_{4 d}^{\text {inst }}\left(\tau_{1}, \cdots, \tau_{n}\right)\right|_{\vec{a}=-\vec{m}-\epsilon_{1} \vec{n}} .
$$

In conjunction with the perturbative part eq. (4.39), this relation (with $\tau_{i}=\tau$ for all $i$ ) shows the equivalence of the $2 \mathrm{~d} / 4 \mathrm{~d}$ partition functions eq. (4.5).

Let us remark that the differential equation for the Nekrasov partition function at the root of Higgs branch, discussed in this paper, is a special case of that studied in [50,51]. From this point of view, the simplification due to the root of Higgs branch would be related to the Nekrasov-Shatashvili limit of the corresponding Hecke algebra [52]. 


\section{Conclusion}

In this paper, we have considered the $\Omega$-background formulation in two dimensions, and applied the localization scheme to obtain the exact formula of the partition function. We have evaluated the one-loop determinant in the two-dimensional $\Omega$-background configuration, and obtained the combinatorial and integral representations of the partition function. We have observed the resultant expression is essentially equivalent to the exact result for the two-dimensional hemisphere. We have then studied the differential equation which the partition function satisfies through the quantization of the classical algebraic geometry. We have considered the vortex worldsheet theory as an application of the exact formula obtained in this paper. We have observed the agreement of the two- and four-dimensional theories at the level of the partition function, by taking the root of Higgs branch.

Let us comment on some possibilities of future works. The first interesting direction is generalization to the three-dimensional theory on $\mathbb{R}^{2} \times S^{1}$, which is the $q$-deformation of the current situation. For such a theory, a similar relation between three- and five-dimensional theories is expected to hold [23-25]. See also $[53,54]$. If we obtain the three-dimensional partition function, we could check the $3 \mathrm{~d} / 5 \mathrm{~d}$ correspondence in the same way as this paper. Furthermore, to see its relation to the $q$-deformed CFT correlation functions, we have to extend our analysis to the quiver gauge theory in two or three dimensions. We can start with, for example, the brane configuration considered in $[48,49]$, and then check the coincidence of the partition functions more explicitly.

\section{Acknowledgments}

The authors are grateful to H. Fuji, Y. Matsuo, and M. Taki for useful comments. The work of MN is supported in part by a Grant-in-Aid for Scientific Research on Innovative Areas "Topological Materials Science" (KAKENHI Grant No. 15H05855) and "Nuclear Matter in Neutron Stars Investigated by Experiments and Astronomical Observations" (KAKENHI Grant No. 15H00841) from the the Ministry of Education, Culture, Sports, Science (MEXT) of Japan. The work of TF and MN is also supported in part by the Japan Society for the Promotion of Science (JSPS) Grant-in-Aid for Scientific Research (KAKENHI Grant No. 25400268) and by the MEXT-Supported Program for the Strategic Research Foundation at Private Universities "Topological Science" (Grant No. S1511006). The work of TK was supported in part by JSPS Grant-in-Aid for Scientific Research (No. 13J04302) from MEXT of Japan.

\section{A Spinor conventions}

Our convention for the anti-symmetric tensors $\epsilon^{\alpha \beta}$ and $\epsilon_{\dot{\alpha} \dot{\beta}}$ is

$$
\epsilon^{12}=\epsilon_{1 \dot{2}}=1, \quad \epsilon^{21}=\epsilon_{\dot{2} \dot{1}}=-1
$$

The $4 \mathrm{~d}$ Weyl spinor indices are raised and lowered as

$$
\lambda^{\alpha}=\epsilon^{\alpha \beta} \lambda_{\beta}, \quad \bar{\lambda}_{\dot{\alpha}}=\epsilon_{\dot{\alpha} \dot{\beta}} \bar{\lambda}^{\dot{\beta}} .
$$


The spinor bilinears without spinor indices are given by

$$
\lambda \psi=\lambda^{\alpha} \psi_{\alpha}, \quad \bar{\lambda} \bar{\psi}=\bar{\lambda}_{\dot{\alpha}} \bar{\psi}^{\dot{\alpha}}, \quad \bar{\lambda} \sigma_{i} \psi=\bar{\lambda}_{\dot{\alpha}}\left(\sigma_{i}\right)^{\dot{\alpha} \beta} \psi_{\beta}, \quad \lambda \bar{\sigma}_{i} \bar{\psi}=\lambda^{\alpha}\left(\bar{\sigma}_{i}\right)_{\alpha \dot{\beta}} \bar{\psi}^{\dot{\beta}} .
$$

Let $\sigma_{i}=\bar{\sigma}_{i}(i=1,2,3)$ be the standard Pauli matrices and

$$
\sigma_{4}=-\bar{\sigma}_{4}=\left(\begin{array}{ll}
i & 0 \\
0 & i
\end{array}\right) .
$$

The rotation generators are defined by

$$
\sigma_{a b}=-\frac{i}{4}\left(\bar{\sigma}_{a} \sigma_{b}-\bar{\sigma}_{a} \sigma_{b}\right), \quad \bar{\sigma}_{a b}=-\frac{i}{4}\left(\sigma_{a} \bar{\sigma}_{b}-\sigma_{a} \bar{\sigma}_{b}\right),
$$

Under the $2 \mathrm{~d}$ rotation $x_{1}+i x_{2} \rightarrow e^{i \theta}\left(x_{1}+i x_{2}\right), 2 \mathrm{~d}$ spinors transform as

$$
\lambda \rightarrow e^{-i \theta \sigma_{12}} \lambda, \quad \bar{\lambda} \rightarrow e^{-i \theta \bar{\sigma}_{12}} \bar{\lambda} .
$$

\section{B Mode expansion around saddle points}

Here we consider the mode expansion around the BPS background Eq (2.48). For notational simplicity, we set $g=1$ and the flavor indices are suppressed in the following. Let us first determine the operators $\Delta_{B}$ and $\Delta_{F}$ appearing in the linearized equations of motion

$$
\Delta_{B} \Phi=0, \quad \Delta_{F} \Psi=0 .
$$

The fermionic part of the action takes the form

$$
\mathcal{L}_{F}=-i\left(\bar{\Psi} \cdot \nabla \Psi+\nabla^{*} \bar{\Psi} \cdot \Psi\right), \quad \Psi=\left(\begin{array}{c}
\bar{\lambda}_{0} \\
\bar{\lambda}_{\bar{z}} \\
\psi_{0} \\
\psi_{\bar{z}}
\end{array}\right), \quad \bar{\Psi}=\left(\begin{array}{c}
\lambda_{0} \\
\lambda_{z} \\
\bar{\psi}_{0} \\
\bar{\psi}_{z}
\end{array}\right)
$$

where $\nabla$ is given by

$$
\nabla \equiv\left(\begin{array}{cccc}
\mathcal{D}_{\xi} & \partial_{z} & \frac{\bar{\phi}}{\sqrt{2}} & 0 \\
-\partial_{\bar{z}} & \mathcal{D}_{\bar{\xi}} & 0 & -\frac{\bar{\phi}}{\sqrt{2}} \\
\frac{\phi}{\sqrt{2}} & 0 & \mathcal{D}_{\bar{\xi}} & \mathcal{D}_{z} \\
0 & -\frac{\bar{\phi}}{\sqrt{2}} & -\mathcal{D}_{\bar{z}} & \mathcal{D}_{\xi}
\end{array}\right)
$$

and $\nabla^{*}$ is the adjoint operator of $\nabla$. The equations of motion for the fermionic fields is $-i \nabla \Psi=0$ and hence

$$
\Delta_{F}=-i \nabla
$$

The bosonic equations of motion can be written in terms of the supersymmetry variations of the fermionic fields:

$$
\left(\begin{array}{c}
-\frac{\delta S}{\delta A_{\bar{\xi}}} \\
\frac{\delta S}{\delta A_{z}} \\
\frac{-i}{\sqrt{2}} \frac{\delta S}{\delta \phi} \\
0
\end{array}\right)=2 \nabla(\mathcal{Q} \Psi), \quad \mathcal{Q} \Psi \equiv\left(\begin{array}{c}
\mathcal{Q} \bar{\lambda}_{0} \\
\mathcal{Q} \bar{\lambda}_{\bar{z}} \\
\mathcal{Q} \psi_{0} \\
\mathcal{Q} \psi_{\bar{z}}
\end{array}\right)
$$


Note that the equation in the forth component is an identity which is automatically satisfied. Since $\mathcal{Q} \Psi=0$ in the BPS background, the linearized version of the bosonic equations of motion (B.5) can be obtained by expanding $\delta \Psi$ in terms of the bosonic fluctuations. It is convenient to impose the following gauge fixing condition for the bosonic fluctuations $\left(\delta A_{\xi}, \delta A_{\bar{z}}, \delta \phi\right)$ :

$$
\left(\partial_{z} \delta A_{\bar{z}}+\mathcal{D}_{\bar{\xi}} \delta A_{\xi}-\frac{i}{2} \delta \phi \bar{\phi}\right)+(\text { c.c. })=0 .
$$

Then, $\mathcal{Q} \Psi$ can be expanded as

$$
\mathcal{Q} \Psi \approx 2\left(\nabla-\mathcal{D}_{\xi}-\mathcal{D}_{\bar{\xi}}\right) \Phi, \quad \Phi \equiv\left(\begin{array}{c}
-\delta A_{\xi} \\
\delta A_{\bar{z}} \\
\frac{-i}{\sqrt{2}} \delta \phi \\
0
\end{array}\right)
$$

where $\approx$ denotes the equality up to the first order in the fluctuation. Note that $\delta \Psi \approx 0$ is the linearized version of the BPS equations (2.41) and (2.42). Eqs. (B.5) and (B.7) imply that the linearized bosonic equations of motion is $\nabla\left(\nabla-\mathcal{D}_{\xi}-\mathcal{D}_{\bar{\xi}}\right) \Phi=0$ and hence

$$
\Delta_{B}=\nabla\left(\nabla-\mathcal{D}_{\xi}-\mathcal{D}_{\bar{\xi}}\right) .
$$

Thus, the eigenequations for the fluctuations are given by

$$
\nabla\left(\nabla-\mathcal{D}_{\xi}-\mathcal{D}_{\bar{\xi}}\right) \Phi=m_{B}^{2} \Phi, \quad \nabla \Psi=i m_{F} \Psi .
$$

Note that $\Phi$ has to satisfy

$$
P \Phi=\Phi, \quad P=\operatorname{diag}(1,1,1,0) .
$$

\section{B.1 Supermultiplets}

Since the background BPS configuration preserve the supersymmetry, the bosonic and fermionic fluctuations form supermultiplets. We can find maps between bosonic and fermionic eigenmodes as follows.

Fermionic eigenmode to bosonic eigenmode. Since the background field satisfy the BPS equation (2.42), the operator $\nabla$ commutes with $\mathcal{D}_{\xi}$ and $\mathcal{D}_{\bar{\xi}}$

$$
\left[\nabla, \mathcal{D}_{\xi}\right]=\left[\nabla, \mathcal{D}_{\bar{\xi}}\right]=0,
$$

and hence they can have simultaneous eigenvectors. Assume that $\Psi$ is a fermionic eigenmode such that

$$
\nabla \Psi=i m_{F} \Psi, \quad \mathcal{D}_{\xi} \Psi=i m_{\xi} \Psi, \quad \mathcal{D}_{\bar{\xi}} \Psi=i m_{\bar{\xi}} \Psi .
$$

Define $\Phi$ by

$$
\Phi=P \Psi .
$$

Since $\Delta_{B} \Psi=m_{F}\left(m_{\xi}+m_{\bar{\xi}}-m_{F}\right)$ and $\left[P, \Delta_{B}\right]=0, \Phi$ is a bosonic eigenmode with eigenvalue $m_{B}^{2}=m_{F}\left(m_{\xi}+m_{\bar{\xi}}-m_{F}\right)$. 
Bosonic eigenmode to fermionic eigenmodes. Assume that $\Phi$ is a bosonic eigenmode with eigenvalues

$$
\nabla\left(\nabla-\mathcal{D}_{\xi}-\mathcal{D}_{\bar{\xi}}\right) \Phi=m_{B}^{2} \Phi, \quad \mathcal{D}_{\xi} \Phi=i m_{\xi} \Phi, \quad \mathcal{D}_{\bar{\xi}} \Phi=i m_{\bar{\xi}} \Phi
$$

Define $\Psi$ by

$$
\Psi=\left(\nabla-\mathcal{D}_{\xi}-\mathcal{D}_{\bar{\xi}}+i m\right) \Phi
$$

This is a fermionic eigenmode with eigenvalue $m_{F}=m$ if $m$ satisfies $m_{B}^{2}=m\left(m_{\xi}+m_{\bar{\xi}}-m\right)$, i.e.,

$$
m=\frac{1}{2}\left[\left(m_{\xi}+m_{\bar{\xi}}\right) \pm \sqrt{\left(m_{\xi}+m_{\bar{\xi}}\right)^{2}-4 m_{B}^{2}}\right] \quad\left(\equiv m_{ \pm}\right)
$$

Thus, we can find two fermionic eigenmodes with eigenvalues $m_{ \pm}$for each generic bosonic eigenmode.

Using the maps eqs. (B.13) and (B.15), we can find several types of supermultiplets. Let us start with the bosonic component. Since $\left[P \nabla, \Delta_{B}\right] \Phi=0$, we can assume that $\Phi$ is an eigenfunction of $P \nabla$ :

$$
P \nabla \Phi=i m \Phi
$$

Generic eigenmodes and ghosts. For a generic eigenmode with $m \neq m_{\bar{\xi}}$, $\Phi$ takes the form

$$
\Phi=\left(\begin{array}{c}
-\delta A_{\xi} \\
\delta A_{\bar{z}} \\
\frac{-i}{\sqrt{2}} \delta \phi \\
0
\end{array}\right), \quad \delta A_{\xi}=i\left(m_{\bar{\xi}}-m\right) \alpha, \quad \delta A_{\bar{z}}=-\partial_{\bar{z}} \alpha, \quad \delta \phi=i \alpha \phi
$$

where $\alpha$ is a function satisfying

$$
\left(-\partial_{z} \partial_{\bar{z}}+\frac{1}{2}|\phi|^{2}\right) \alpha=\left|m-m_{\xi}\right|^{2} \alpha .
$$

This is actually an eigenmode of $\nabla$ with eigenvalue $m$. We can show that contributions from these modes are canceled by those from the ghosts for the gauge fixing condition eq. (B.6).

Long multiplets. From the explicit form of $\nabla$, we can show that $\delta A_{\xi}=0$ for any eigenmode of $P \nabla$ with eigenvalue $m=m_{\bar{\xi}}$. Then, it follows that the map eq. (B.15) gives two fermionic eigenmodes $\Psi_{ \pm}$such that $P \Psi_{ \pm} \propto \Phi$. Thus, a generic supermultiplet of this type consists of one bosonic and two fermionic eigenmodes. Since the eigenvalues are related by $m_{B}=m_{+} m_{-}$, these multiplets do not contribute to the ratio of the one-loop determinants in eq. (2.36). 
Short multiplets. The supermultiplets which have non-trivial contribution are short multiplets which can be obtained by requiring that $\Phi$ is an eigenmode of $\nabla$ with eigenvalue $m=m_{\bar{\xi}}$ :

$$
\nabla \Phi=i m_{\bar{\xi}} \Phi
$$

This is a bosonic eigenmode with eigenvalue $\Delta_{B}=m_{\xi} m_{\bar{\xi}}$ and the map eq. (B.15) gives only one partner fermionic mode $\Psi \propto \Phi$ with eigenvalue $\Delta_{F}=m_{\bar{\xi}}$. Thus the ratio of the determinant reduces to

$$
\frac{\operatorname{det} \Delta_{B}}{\operatorname{det} \Delta_{F}}=\prod_{\text {short }} \frac{1}{m_{\xi}}=\left.\frac{1}{\operatorname{det}\left(-i \mathcal{D}_{\xi}\right)}\right|_{\text {short }} .
$$

Since $\delta A_{\xi}=0$, the eigenmode equation (B.20) reduces to

$$
\left(\begin{array}{cc}
\partial_{z} & \frac{\bar{\phi}}{\sqrt{2}} \\
-\frac{\bar{\phi}}{\sqrt{2}} & -\mathcal{D}_{\bar{z}}
\end{array}\right)\left(\begin{array}{c}
\delta A_{\bar{z}} \\
\frac{-i}{\sqrt{2}} \delta \phi
\end{array}\right)=0 .
$$

This is the linearized version of the BPS vortex equation (2.41), whose solution can be formally written as

$$
\delta A_{\bar{z}}=-\frac{i}{2} \partial_{\bar{z}} \delta \omega, \quad \delta \phi=\sqrt{r} e^{-\frac{1}{2} \omega}\left[\delta h-\frac{1}{2} \delta \omega h(z)\right],
$$

where $\omega$ and $h(z)$ are the function appearing in the background solution eq. (2.43) and $\delta \omega$ is the function satisfying

$$
\left(-\partial_{z} \partial_{\bar{z}}+\frac{1}{2}|\phi|^{2}\right) \delta \omega=e^{-\frac{1}{2} \omega} \bar{\phi} \delta h(z) .
$$

This solution is a eigenfunction of $\mathcal{D}_{\xi}$, if one of the holomorphic functions $\delta h(z)=$ $\left(\delta h_{1}, \delta h_{2}, \cdots, \delta h_{N}\right)$ is non-zero and takes the form

$$
\delta h_{b}(z)=z^{l} \exp i\left(\frac{n_{3} x_{3}}{R_{3}}+\frac{n_{4} x_{4}}{R_{4}}\right) .
$$

For such a eigenmode, the eigenvalue $m_{\xi}$ is given by

$$
m_{\xi}=-\frac{i}{2}\left(\frac{n_{3}}{R_{3}}-i \frac{n_{4}}{R_{4}}\right)+\left(m_{b}-m_{a}\right)+(l-k) \epsilon,
$$

where we have assumed $\phi_{a} \propto z^{k}$ in the BPS background.

\section{B.2 Normalizability}

In the above discussion, we did not take into account the normalizability of the eigenmodes. The eigenmodes eq. (B.23) with $l \geq k$ are non-normalizable since the boundary condition $\omega \rightarrow \log |z|^{2 k}$ implies that

$$
\sqrt{r} e^{-\frac{1}{2} \omega} \delta h(z) \approx \sqrt{r} z^{l} /|z|^{k} \rightarrow \infty \quad(\text { for large }|z|) .
$$


We can solve this problem by making all the modes normalizable in the following way. Since the partition function is invariant under any deformation of $\mathcal{Q} V$, we can freely change the normalization factor of the kinetic terms in $\mathcal{Q} V$. Let us consider the following deformation of $V$

$$
\begin{aligned}
V= & \frac{1}{e^{2}}\left(\lambda_{z} \overline{\delta \lambda_{z}}+\lambda_{0} \overline{\delta \lambda_{0}}+\bar{\lambda}_{0} \overline{\delta \bar{\lambda}_{0}}+\bar{\lambda}_{\bar{z}} \overline{\delta \bar{\lambda}_{\bar{z}}}\right) \\
& +e^{-\alpha}\left[\psi_{0}^{a} \overline{\delta \psi_{0}^{a}}+\psi_{\bar{z}}^{a} \overline{\delta \psi_{\bar{z}}^{a}}+\bar{\psi}_{z}^{a} \overline{\delta \bar{\psi}_{z}^{a}}+\bar{\psi}_{0}^{a} \overline{\delta \bar{\psi}_{0}^{a}}+i\left(\lambda_{0}+\bar{\lambda}_{0}\right)\left(\left|\phi_{a}\right|^{2}-r\right)\right],
\end{aligned}
$$

where $\alpha$ is a function of $|z|^{2}$ such that $\alpha \rightarrow \infty$ for large $|z|$. By the field redefinition

$$
\phi=e^{\frac{1}{2} \alpha} \hat{\phi}, \quad \psi=e^{\frac{1}{2} \alpha} \hat{\psi}, \quad A_{i} d x^{i}=\hat{A}_{i} d x^{i}+\frac{d \alpha}{d|z|^{2}}\left(x_{1} e_{2}-x_{2} e_{1}\right)
$$

the action takes the original form with the following position-dependent FI parameter

$$
\hat{r}=r e^{-\alpha}+\frac{2}{e^{2}} \partial_{z} \partial_{\bar{z}} \alpha .
$$

Since boundary condition for the profile function $\omega$ does not change, the asymptotic form of the eigenmode becomes

$$
\left.\sqrt{\hat{r}} e^{-\frac{1}{2} \omega} \delta h(z) \approx \sqrt{r} e^{-\frac{1}{2} \alpha} z^{l} /|z|^{k} \rightarrow 0 \quad \text { (for large }|z|\right),
$$

and hence all the eigenmodes become normalizable if the function $\alpha$ is chosen so that $e^{-\frac{1}{2} \alpha}$ decreases faster than polynomials. We can also show that this deformation eliminates all the unpaired fermionic eigenmodes which can exist when there are negatively charged chiral multiplets (e.g. anti-fundamental fields).

Open Access. This article is distributed under the terms of the Creative Commons Attribution License (CC-BY 4.0), which permits any use, distribution and reproduction in any medium, provided the original author(s) and source are credited.

\section{References}

[1] V. Pestun, Localization of gauge theory on a four-sphere and supersymmetric Wilson loops, Commun. Math. Phys. 313 (2012) 71 [arXiv:0712.2824] [InSPIRE].

[2] N.A. Nekrasov, Seiberg-Witten prepotential from instanton counting, Adv. Theor. Math. Phys. 7 (2003) 831 [hep-th/0206161] [INSPIRE].

[3] S. Shadchin, On F-term contribution to effective action, JHEP 08 (2007) 052 [hep-th/0611278] [INSPIRE].

[4] T. Dimofte, S. Gukov and L. Hollands, Vortex Counting and Lagrangian 3-manifolds, Lett. Math. Phys. 98 (2011) 225 [arXiv:1006.0977] [INSPIRE].

[5] Y. Yoshida, Localization of Vortex Partition Functions in $\mathcal{N}=(2,2)$ Super Yang-Mills theory, arXiv:1101.0872 [INSPIRE].

[6] G. Bonelli, A. Tanzini and J. Zhao, Vertices, Vortices and Interacting Surface Operators, JHEP 06 (2012) 178 [arXiv:1102.0184] [INSPIRE]. 
[7] G. Bonelli, A. Tanzini and J. Zhao, The Liouville side of the Vortex, JHEP 09 (2011) 096 [arXiv: 1107.2787] [INSPIRE].

[8] T. Dimofte, D. Gaiotto and S. Gukov, 3-Manifolds and 3d Indices, Adv. Theor. Math. Phys. 17 (2013) 975 [arXiv:1112.5179] [INSPIRE].

[9] T. Fujimori, T. Kimura, M. Nitta and K. Ohashi, Vortex counting from field theory, JHEP 06 (2012) 028 [arXiv:1204.1968] [INSPIRE].

[10] A. Hanany and D. Tong, Vortices, instantons and branes, JHEP 07 (2003) 037 [hep-th/0306150] [INSPIRE].

[11] R. Auzzi, S. Bolognesi, J. Evslin, K. Konishi and A. Yung, NonAbelian superconductors: vortices and confinement in $N=2$ SQCD, Nucl. Phys. B 673 (2003) 187 [hep-th/0307287] [INSPIRE].

[12] D. Tong, TASI lectures on solitons: Instantons, monopoles, vortices and kinks, hep-th/0509216 [INSPIRE].

[13] M. Eto, Y. Isozumi, M. Nitta, K. Ohashi and N. Sakai, Moduli space of non-Abelian vortices, Phys. Rev. Lett. 96 (2006) 161601 [hep-th/0511088] [INSPIRE].

[14] M. Eto et al., Non-Abelian Vortices of Higher Winding Numbers, Phys. Rev. D 74 (2006) 065021 [hep-th/0607070] [INSPIRE].

[15] M. Eto, Y. Isozumi, M. Nitta, K. Ohashi and N. Sakai, Solitons in the Higgs phase: The Moduli matrix approach, J. Phys. A 39 (2006) R315 [hep-th/0602170] [INSPIRE].

[16] M. Shifman and A. Yung, Supersymmetric Solitons and How They Help Us Understand Non-Abelian Gauge Theories, Rev. Mod. Phys. 79 (2007) 1139 [hep-th/0703267] [InSPIRE].

[17] D. Tong, Quantum Vortex Strings: A Review, Annals Phys. 324 (2009) 30 [arXiv:0809.5060] [INSPIRE].

[18] N.A. Nekrasov and S.L. Shatashvili, Quantization of Integrable Systems and Four Dimensional Gauge Theories, arXiv:0908.4052 [INSPIRE].

[19] N. Nekrasov and V. Pestun, Seiberg-Witten geometry of four dimensional $N=2$ quiver gauge theories, arXiv:1211.2240 [INSPIRE].

[20] N. Nekrasov, V. Pestun and S. Shatashvili, Quantum geometry and quiver gauge theories, arXiv:1312.6689 [INSPIRE].

[21] N. Dorey, The BPS spectra of two-dimensional supersymmetric gauge theories with twisted mass terms, JHEP 11 (1998) 005 [hep-th/9806056] [INSPIRE].

[22] N. Dorey, T.J. Hollowood and D. Tong, The BPS spectra of gauge theories in two-dimensions and four-dimensions, JHEP 05 (1999) 006 [hep-th/9902134] [INSPIRE].

[23] M. Aganagic, N. Haouzi, C. Kozcaz and S. Shakirov, Gauge/Liouville Triality, arXiv:1309.1687 [INSPIRE].

[24] M. Aganagic, N. Haouzi and S. Shakirov, $A_{n}$-Triality, arXiv:1403.3657 [INSPIRE].

[25] M. Aganagic and N. Haouzi, ADE Little String Theory on a Riemann Surface (and Triality), arXiv: 1506.04183 [INSPIRE].

[26] H.-Y. Chen and H.-Y. Chen, Heterotic Surface Defects and Dualities from 2d/4d Indices, JHEP 10 (2014) 004 [arXiv: 1407.4587] [InSPIRE]. 
[27] H.-Y. Chen and T.-H. Tsai, On Higgs Branch Localization of Seiberg-Witten Theories on Ellipsoid, arXiv: 1506.04390 [INSPIRE].

[28] Y. Pan and W. Peelaers, Ellipsoid partition function from Seiberg-Witten monopoles, JHEP 10 (2015) 183 [arXiv:1508.07329] [INSPIRE].

[29] A. Hanany and D. Tong, Vortex strings and four-dimensional gauge dynamics, JHEP 04 (2004) 066 [hep-th/0403158] [INSPIRE].

[30] M. Shifman and A. Yung, NonAbelian string junctions as confined monopoles, Phys. Rev. D 70 (2004) 045004 [hep-th/0403149] [inSPIRE].

[31] S. Sugishita and S. Terashima, Exact Results in Supersymmetric Field Theories on Manifolds with Boundaries, JHEP 11 (2013) 021 [arXiv:1308.1973] [INSPIRE].

[32] D. Honda and T. Okuda, Exact results for boundaries and domain walls in $2 d$ supersymmetric theories, JHEP 09 (2015) 140 [arXiv:1308.2217] [INSPIRE].

[33] K. Hori and M. Romo, Exact Results In Two-Dimensional (2,2) Supersymmetric Gauge Theories With Boundary, arXiv:1308.2438 [INSPIRE].

[34] K. Hori and C. Vafa, Mirror symmetry, hep-th/0002222 [INSPIRE].

[35] E.K. Sklyanin, The Quantum Toda Chain, Lect. Notes Phys. 226 (1985) 196 [InSPIRE].

[36] M. Eto et al., Fractional Vortices and Lumps, Phys. Rev. D 80 (2009) 045018 [arXiv:0905.3540] [INSPIRE].

[37] T. Kimura and M. Nitta, Vortices on Orbifolds, JHEP 09 (2011) 118 [arXiv:1108.3563] [INSPIRE].

[38] J. Zhao, Orbifold Vortex and Super Liouville Theory, arXiv:1111.7095 [INSPIRE].

[39] A. D'Adda, A.C. Davis, P. Di Vecchia and P. Salomonson, An Effective Action for the Supersymmetric $C P^{(N-1)}$ Model, Nucl. Phys. B 222 (1983) 45 [InSPIRE].

[40] S. Cecotti and C. Vafa, On classification of $N=2$ supersymmetric theories, Commun. Math. Phys. 158 (1993) 569 [hep-th/9211097] [INSPIRE].

[41] E. Witten, Phases of $N=2$ theories in two-dimensions, Nucl. Phys. B 403 (1993) 159 [hep-th/9301042] [INSPIRE].

[42] D.R. Morrison and M.R. Plesser, Summing the instantons: Quantum cohomology and mirror symmetry in toric varieties, Nucl. Phys. B 440 (1995) 279 [hep-th/9412236] [INSPIRE].

[43] M. Eto, Y. Isozumi, M. Nitta, K. Ohashi and N. Sakai, Instantons in the Higgs phase, Phys. Rev. D 72 (2005) 025011 [hep-th/0412048] [INSPIRE].

[44] T. Fujimori, M. Nitta, K. Ohta, N. Sakai and M. Yamazaki, Intersecting Solitons, Amoeba and Tropical Geometry, Phys. Rev. D 78 (2008) 105004 [arXiv:0805.1194] [InSPIRE].

[45] E. Witten, Solutions of four-dimensional field theories via M-theory, Nucl. Phys. B 500 (1997) 3 [hep-th/9703166] [INSPIRE].

[46] D. Tong, Monopoles in the Higgs phase, Phys. Rev. D 69 (2004) 065003 [hep-th/0307302] [INSPIRE].

[47] M. Nitta and W. Vinci, Non-Abelian Monopoles in the Higgs Phase, Nucl. Phys. B 848 (2011) 121 [arXiv: 1012.4057] [INSPIRE]. 
[48] N. Dorey, S. Lee and T.J. Hollowood, Quantization of Integrable Systems and a $2 d / 4 d$ Duality, JHEP 10 (2011) 077 [arXiv:1103.5726] [INSPIRE].

[49] H.-Y. Chen, N. Dorey, T.J. Hollowood and S. Lee, A New 2d/4d Duality via Integrability, JHEP 09 (2011) 040 [arXiv: 1104.3021] [INSPIRE].

[50] S. Kanno, Y. Matsuo and H. Zhang, Virasoro constraint for Nekrasov instanton partition function, JHEP 10 (2012) 097 [arXiv: 1207.5658] [INSPIRE].

[51] S. Kanno, Y. Matsuo and H. Zhang, Extended Conformal Symmetry and Recursion Formulae for Nekrasov Partition Function, JHEP 08 (2013) 028 [arXiv:1306.1523] [INSPIRE].

[52] J.-E. Bourgine, Spherical Hecke algebra in the Nekrasov-Shatashvili limit, JHEP 01 (2015) 114 [arXiv: 1407.8341] [INSPIRE].

[53] F. Nieri, S. Pasquetti and F. Passerini, $3 d$ and 5d Gauge Theory Partition Functions as q-deformed CFT Correlators, Lett. Math. Phys. 105 (2015) 109 [arXiv:1303.2626] [INSPIRE].

[54] F. Nieri, S. Pasquetti, F. Passerini and A. Torrielli, 5D partition functions, q-Virasoro systems and integrable spin-chains, JHEP 12 (2014) 040 [arXiv:1312.1294] [INSPIRE]. 